\title{
Fibroblast growth factor signals regulate a wave of Hedgehog activation that is essential for coronary vascular development
}

\author{
Kory J. Lavine, ${ }^{1}$ Andrew C. White, ${ }^{1}$ Changwon Park, ${ }^{2}$ Craig S. Smith, ${ }^{1}$ Kyunghee Choi, ${ }^{2}$ \\ Fanxin Long, ${ }^{1,3}$ Chi-chung Hui, ${ }^{4}$ and David M. Ornitz ${ }^{1,5}$ \\ ${ }^{1}$ Department of Molecular Biology and Pharmacology, ${ }^{2}$ Department of Pathology and Immunology, and ${ }^{3}$ Department of \\ Internal Medicine, Washington University Medical School, St. Louis, Missouri 63110, USA; ${ }^{4}$ Program in Developmental \\ Biology, The Hospital for Sick Children, and Department of Molecular and Medical Genetics, University of Toronto, \\ Toronto, Ontario M5G 1X8 Canada
}

Myocardial infarction and ischemic heart disease are the leading cause of death in the industrial world. Therapies employed for treating these diseases are aimed at promoting increased blood flow to cardiac tissue. Pharmacological induction of new coronary growth has recently been explored, however, clinical trials with known proangiogenic factors have been disappointing. To identify novel therapeutic targets, we have explored signaling pathways that govern embryonic coronary development. Using a combination of genetically engineered mice and an organ culture system, we identified novel roles for fibroblast growth factor (FGF) and Hedgehog $(\mathrm{HH})$ signaling in coronary vascular development. We show that FGF signals promote coronary growth indirectly by signaling to the cardiomyoblast through redundant function of Fgfr1 and Fgfr2. Myocardial FGF signaling triggers a wave of $\mathrm{HH}$ activation that is essential for vascular endothelial growth factor (Vegf)-A, Vegf-B, Vegf-C, and angiopoietin-2 (Ang2) expression. We demonstrate that HH is necessary for coronary vascular development and activation of $\mathrm{HH}$ signaling is sufficient to promote coronary growth and to rescue coronary defects due to loss of FGF signaling. These studies implicate HH signaling as an essential regulator of coronary vascular development and as a potential therapeutic target for coronary neovascularization. Consistent with this, activation of $\mathrm{HH}$ signaling in the adult heart leads to an increase in coronary vessel density.

[Keywords: Fibroblast growth factor (FGF); Hedgehog (HH); heart development; coronary vascular development; coronary neoangiogenesis]

Supplemental material is available at http://www.genesdev.org.

Received January 20, 2006; revised version accepted April 12, 2006.

Insufficient myocardial vascularization and/or perfusion are paramount to the development of ischemic heart disease and heart failure. Recently, it has been demonstrated that expression of molecules that function during embryonic vascular development can lead to formation of new coronary arteries in the adult myocardium. Overexpression of fibroblast growth factor-2 (Fgf2), vascular endothelial growth factor-A (Vegf-A), and angiopoietin-2 (Ang2) in the adult myocardium leads to significant increases in coronary artery number (Landau et al. 1995; Uchida et al. 1995; Rajanayagam et al. 2000; Visconti et al. 2002; House et al. 2003; Syed et al. 2004; Tammela et

${ }^{4}$ Corresponding author.

E-MAIL dornitz@wustl.edu; FAX (314) 362-7058.

Article is online at http://www.genesdev.org/cgi/doi/10.1101/gad.1411406. al. 2005). The effects of Fgf2 and Vegf-A in the adult heart have been intensively investigated, and are candidates for treatment of ischemic heart disease (Scheinowitz et al. 1997; Syed et al. 2004). However, despite their ability to promote new blood vessel growth in both normal and ischemic hearts, clinical trails utilizing either protein or gene therapy have been disappointing (Henry et al. 2000; Losordo et al. 2002; Simons et al. 2002; Syed et al. 2004).

One approach to identify new molecules that promote coronary vascular growth and to develop novel or improved therapeutic agents is to investigate how the coronary vascular system develops. Several FGFs (Fgf1, Fgf2, $F g f 9, F g f 16$, and Fgf20), VEGFs (Vegf-A and Vegf-B), and angiopoietins (Ang1 and Ang2) are expressed in the embryonic heart during coronary formation (Tomanek et al. 
2002; Ward and Dumont 2002; Detillieux et al. 2003; Lavine et al. 2005). However, little is known about whether or how these molecules orchestrate the development of the coronary vascular system. Elucidation of the mechanism by which these molecules control coronary development and identification of new factors that regulate their expression should provide important information to guide the development of novel therapeutics.

In contrast to the scarcity of information about signaling pathways controlling coronary vascular development, many of the morphological events involved in its formation have been well described, especially in avian systems (Morabito et al. 2002; Reese et al. 2002; Wada et al. 2003). Prior to stage 14 in the chick (embryonic day 9.5 [E9.5] in the mouse), the heart consists of two layers, an outer myocardial layer and inner endocardial layer. At stage 18 in the chick (E10.5 in the mouse), the third cardiac layer, the epicardium, migrates to and envelopes the embryonic heart. Epicardial progenitors are derived from the proepicardial organ (an epithelium associated with the septum transversum), which later migrates to the developing heart.

Initial studies in avian systems demonstrated that the proepicardial organ and the epicardium are required for coronary development, as removal of either structure severely perturbs coronary vessel formation /Gittenbergerde Groot et al. 2000). More recently, genetic analysis in the mouse has confirmed this observation. Removal of genes required for formation (GATA4) and migration or attachment (VCAM, $\alpha 4 \beta 1$ integrin) of epicardial precursors to the myocardium leads to severe defects in coronary development (Kwee et al. 1995; Yang et al. 1995; Watt et al. 2004). In addition, deletion of genes necessary for survival and/or integrity (p300, WT1) of the epicardium lead to similar phenotypes (Davies et al. 1999; Moore et al. 1999; Shikama et al. 2003; Shao et al. 2005).

It has been proposed that the epicardium physically contributes vascular cell types to the heart through an epithelial mesenchymal transformation (EMT). This hypothesis is supported by lineage analysis of the proepicardial organ in chick and quail models demonstrating that cells contained within the proepicardial organ give rise to perivascular fibroblasts, vascular smooth muscle, and endothelial cells of the coronary vessels (Mikawa and Fischman 1992; Mikawa and Gourdie 1996; PerezPomares et al. 2002). However, both cell labeling and genetic lineage analysis of the epicardium revealed contributions to only perivascular fibroblast and smooth muscle cell lineages but not the coronary endothelial cell lineage (Dettman et al. 1998; Vrancken Peeters et al. 1999; Merki et al. 2005).

Together these lineage studies suggest that the proepicardial organ contains a mixed pool of vascular smooth muscle/fibroblast progenitors and endothelial progenitors, both of which migrate to the heart. Upon reaching the heart, these two progenitors segregate such that vascular smooth muscle cell/fibroblast progenitors are resident within the epicardium while endothelial progenitors occupy another space. This notion is supported by work showing that these two lineages can be indepen- dently identified by immunohistochemical analysis in the migrating proepicardial organ (Dettman et al. 1998).

An alternative explanation for why the epicardium is essential for coronary development is that the epicardium acts as a signaling center. Support for this hypothesis stems from the observation that the epicardium promotes cardiomyoblast proliferation by secreting mitogens (Chen et al. 2002; Stuckmann et al. 2003). Interestingly, not only is retinoic acid (RA) signaling in the epicardium necessary for the secretion of such mitogens but also it is required for coronary development (Sucov et al. 1994; Merki et al. 2005).

Previously we identified Fgf9 as a RA-regulated epicardially derived mitogen essential for cardiomyoblast proliferation (Lavine et al. 2005). From this observation and extensive studies showing that FGF signaling can promote vascular formation and growth (Seghezzi et al. 1998; Auguste et al. 2003; Kanda et al. 2004), we hypothesized that FGFs, such as Fgf9, may also regulate coronary development.

Here, we present evidence that the epicardium acts as a signaling center for coronary development. We demonstrate that epicardial and endocardial sources of FGF ligands control coronary development by signaling to the cardiomyoblast through redundant function of FGF receptors 1 and 2. Myocardial FGF signaling regulates coronary vascular development by triggering a wave of Hedgehog $(\mathrm{HH})$ activation that progresses from the atrial-ventricular groove to the apex of the ventricles. $\mathrm{HH}$ signaling in turn induces the expression of Vegf-A, Vegf$B$, Vegf-C, and Ang2, resulting in the formation of the coronary vascular plexus. We further demonstrate that activation of $\mathrm{HH}$ signaling can rescue the coronary defects seen in hearts lacking myocardial FGF signaling and that Vegf and Ang2 can rescue coronary defects due to lack of $\mathrm{HH}$ signaling. Finally, we show that activation of $\mathrm{HH}$ signaling in the adult myocardium promotes coronary neovascularization, implicating $\mathrm{HH}$ signaling as a novel pharmacological target to increase coronary vessel density and perfusion.

\section{Results \\ Spatiotemporal development of the mouse coronary vascular system}

To assess the normal progression of coronary development, we stained E11.5-E13.5 hearts with an antibody against PECAM to visualize the developing coronary vasculature. Beginning at E11.5, blood vessels emerged in the atrial-ventricular groove (Fig. 1A). Staining of E12.5 hearts revealed the presence of a forming vascular network originating from the atrial-ventricular groove and extending toward the apices of the left and right ventricle (Fig. 1B). By E13.5, the vascular network reached the ventricular apices and covered the entire ventricle (Fig. 1C).

Histological analysis demonstrated blood vessels growing in the subepicardial space (Fig. 1G-I). Interestingly, the presence of vascular endothelium within the 


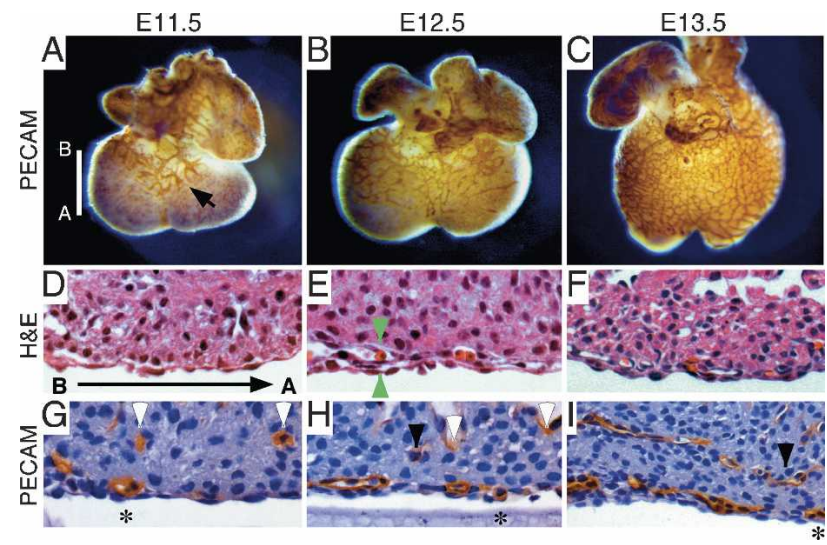

Figure 1. Spatiotemporal development of coronary blood vessels. $(A-C)$ Whole-mount PECAM staining of E11.5, E12.5, and E13.5 hearts. (A) At E11.5 coronary vessels emerge from the atrial-ventricular groove (arrow). $(B, C)$ Between E12.5 and E13.5, coronary vessels extend in a wave-like pattern emanating from the atrial-ventricular and interventricular groove and extend toward the ventricular apices. $(D-F)$ Histology at E11.5, E12.5, and E13.5 demonstrating formation of the subepicardial mesenchyme at E12.5 (E) and vessel-like structures within the subepicardial space at E13.5 $(F)$. Green arrowheads denote location of the subepicardial mesenchyme. $(G-I)$ Histological sections of PECAM-stained hearts demonstrating the presence of blood vessels growing within the subepicardial space (asterisk) and within the myocardial wall (black arrowhead). The asterisk and arrow mark the position of the most distal subepicardial and intramyocardial blood vessel, respectively. Open arrowhead denotes endocardial PECAM staining. Magnification: $A-C, 25 \times$; $D-E, G-H, 400 \times ; F, I, 200 \times$. Bar in $A$ and corresponding arrow in $D$ represent orientation and position of histological sections. (B) Base of ventricle; (A) apex of ventricle.

subepicardial space was coincident with the appearance of subepicardial mesenchyme (Fig. 1E,H), suggesting that coronary vessel growth and epicardial EMT may be linked processes. In addition to subepicardial blood vessels, a second population of blood vessels was detected within the myocardium. Similar to subepicardial blood vessels, these vessels also underwent a spatiotemporal pattern of growth emerging from the atrial-ventricular groove at E11.5 and extending apically to cover much of the ventricle by E13.5 (Fig. 1G-I).

\section{Fgf9 is required for coronary development}

To determine whether Fgf9 is necessary for coronary development, we visualized the coronary vasculature of $\mathrm{Fgf9}^{-/}$embryonic hearts using PECAM staining (Fig. 2A-C). Analysis at E13.5 demonstrated that while littermate control hearts developed a vascular plexus that encased the entire ventricle, the vascular plexus in $\mathrm{FgfO}^{-/-}$ hearts failed to fully extend to the apices of the heart (Fig. 2A,B). Quantitation of the percentage of the ventricle covered by blood vessels revealed that littermate control and $\mathrm{FgfO}^{-/-}$hearts had $95 \pm 1 \%$ and $80 \pm 2 \%$ of the ventricle covered by blood vessels, respectively $(p<0.001)$ (Fig. 2C).
Histological analysis of $\mathrm{Fg} \mathrm{fO}^{-/-}$hearts revealed the presence of an intact epicardium at E11.5 (Fig. 2D,G) and normal expression of the epicardial marker WT-1 (data not shown). In contrast, at E12.5, impairment in the formation of the subepicardial mesenchyme was evident in $\mathrm{Fgf9}^{-/-}$hearts compared with littermate controls (Fig. 2E,H). Further analysis at E13.5 demonstrated that growth of both the subepicardial blood vessels and the vessels growing within the myocardium were delayed in FgfO- $^{--}$hearts (Fig. 2F,I).

\section{FGF signaling to endothelial cells is dispensable for coronary development}

Potential mechanisms by which FGF signaling promotes coronary development include direct signaling to the coronary endothelial cell or indirect signaling to another cell type. Given the enormity of previous work implicating the endothelial cell as a target for proangiogenic FGF signaling and expression of FGFR1 and FGFR2 in endothelial cells (Seghezzi et al. 1998; Auguste et al. 2003; Kanda et al. 2004), we hypothesized that FGF9 signaled to endothelial cells.

To test this hypothesis, we conditionally inactivated both Fgfr1 and Fgfr2 in endothelial cells using either Tie1-cre (Gustafsson et al. 2001) or Flk1-cre (Motoike et al. 2003). To our surprise, deletion of both Fgfr1 and Fgfr2 in endothelial cells did not impair coronary vessel devel-

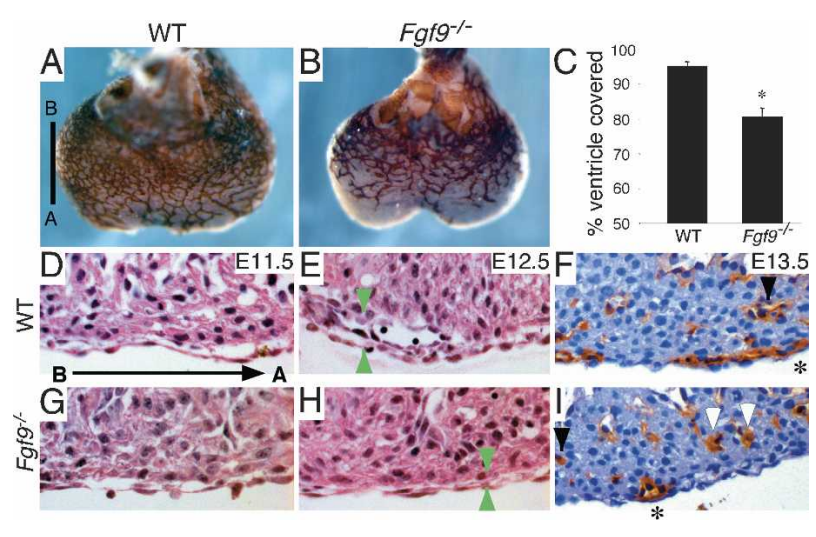

Figure 2. Fgf9 regulates coronary vascular development. $(A, B)$ Whole-mount PECAM staining of E13.5 control $(A)$ and $F g f 9^{-/}$ (B) hearts. Fgf9- ${ }^{-/}$hearts display defects in growth of the coronary plexus. $(C)$ Quantitation of the percentage of the ventricle covered by blood vessels in control and $\mathrm{FgF}^{-1-}$ hearts. Asterisk indicates statistically significant difference compared with controls $(p<0.01)$. $(D-I)$ Histology of control $(D-F)$ and $F_{g f 9^{-/-}}(G-I)$ hearts demonstrating defects in formation of the subepicardial mesenchyme (green arrowheads) in $\mathrm{FgfO}^{-/-}(H)$ compared with controls $(E)$ at E12.5. PECAM-stained section of control $(F)$ and $\mathrm{FgfO}^{-1-}(I)$ hearts showing failure of both subepicardial (asterisk) and intramyocardial (black arrowhead) vessel growth at E13.5. The asterisk and arrowhead mark the position of the most distal subepicardial and intramyocardial blood vessel, respectively. Open arrowhead denotes endocardial PECAM staining. Magnification: $A, C, 25 \times ; D, E, G, H, 400 \times ; F, I, 200 \times$. Bar in $A$ and corresponding arrow in $D$ represent orientation and position of histological sections. (B) Base of ventricle; (A) apex of ventricle. 
opment (Fig. 3A,B,E,F). To assess whether the Tie1-cre and Flk1-cre lines could promote efficient recombination in coronary endothelial cells, we bred the Rosa26LacZ reporter (Soriano 1999) into our Fgfr1/2 double conditional knockout (DCKO) lines. LacZ staining of E13.5 and E16.5 Fgfr1/2 ${ }^{\text {Tie1-cre }}$ (Fig. 3C,D) and Fgfr1/2 Flk1-cre (Fig. 3G,H) DCKO hearts revealed that nearly all endothelial cells in the heart (endocardium and coronary endothelium) had undergone Cre-mediated recombination. Together these data indicate that Cre-mediated recombination had occurred in endothelial cells and that endothelial cells lacking both Fgfr1 and Fgfr2 can contribute to not only the vascular plexus (E13.5) but also mature coronary vessels (E16.5).

\section{FGF signaling to the cardiomyoblast is essential} for coronary development

Since FGF signaling to coronary endothelial cells was not required for coronary development, we considered other potential targets of FGF9. Previously, we identified the cardiomyoblast as the target of FGF9 in the context of myocardial proliferation (Lavine et al. 2005). Given the ability of the cardiomyoblast to receive FGF9 signals, we postulated that the myocardium may also be the target of FGF9 in the context of coronary vascular development.

To test this possibility, we examined embryos that lacked Fgfr1 and Fgfr2 in the embryonic myocardium (Fgfr1/2 Mlc2v-cre DCKO). These embryos are devoid of FGF signaling in the ventricular cardiomyoblast, as previously described (Lavine et al. 2005). PECAM staining of E11.5-E13.5 Fgfr1/2 ${ }^{\text {Mlc2v-cre }}$ DCKO hearts revealed impaired coronary vascular development similar to that of $\mathrm{Fgf9}^{-/-}$hearts (Fig. 4A-F). Comparison of control and Fgfr1/2 ${ }^{\text {Mlc2v-cre }}$ DCKO hearts at E13.5 demonstrated that while control hearts contained a vascular plexus that enclosed the ventricle, the vascular plexus of Fgfr1/ $2^{\text {Mlc2v-cre }}$ DCKO failed to extend apically and was unable to fully encase the heart (Fig. 4C,F). Quantitation of the percentage of the ventricle covered by blood vessels at E13.5 revealed control and Fgfr1/2 ${ }^{\text {Mlc2v-cre }}$ DCKO hearts had $91 \pm 1 \%$ and $77 \pm 2 \%$ of the ventricle covered by blood vessels, respectively $(p<0.001)$ (Fig. 4M). Hearts lacking either Fgfr1 or Fgfr2 in the myocardium were indistinguishable from controls (data not shown).

Examination at earlier stages of coronary development (E11.5 and E12.5) showed that blood vessels emerged normally from the atrial-ventricular groove of both control and Fgfr1/2 ${ }^{\text {Mlc2v-cre }}$ DCKO hearts at E11.5 (Fig. 4A,D). However, by E12.5, Fgfr1/2 ${ }^{\text {Mlc2v-cre }}$ DCKO hearts displayed impaired growth of the vascular plexus (Fig. $4 \mathrm{~B}, \mathrm{E})$. Quantitation of the percentage of the ventricle covered by blood vessels at E12.5 demonstrated that control and Fgfr1/2 ${ }^{\text {Mlc } 2 \text {-cre }}$ DCKO hearts had $74 \pm 2 \%$ and $52 \pm 4 \%$ of the ventricle covered by blood vessels, respectively $(p<0.001)$ (Fig. 4M).

Similar to $\mathrm{FgfO}^{-/-}$hearts, histological analysis of Fgfr1/ $2^{\text {MIc2v-cre }}$ DCKO hearts revealed the presence of an intact epicardium at E11.5 (Fig. 4G,J) and normal expression of

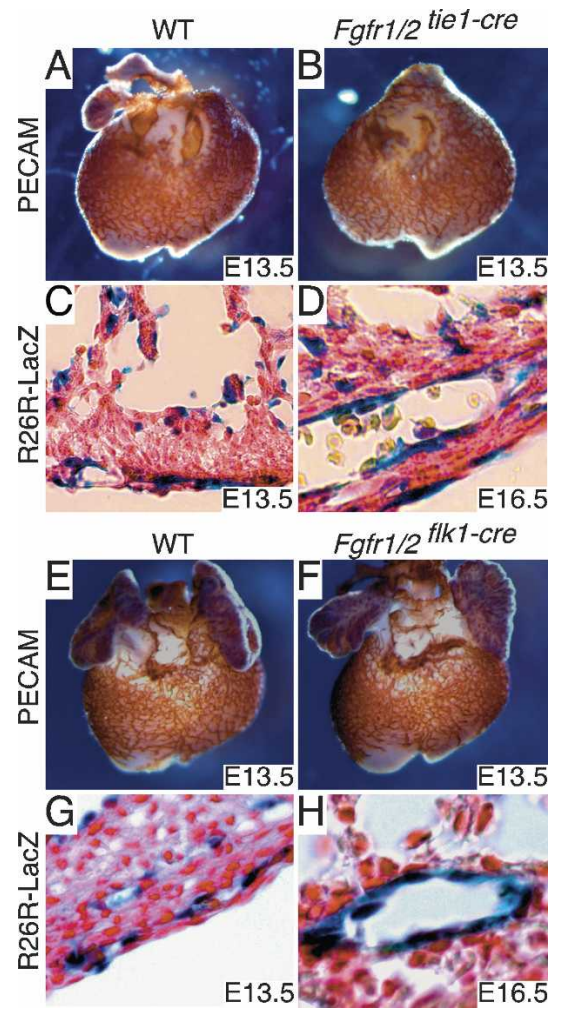

Figure 3. FGF signaling to endothelial cells is not required for coronary vascular development. $(A-D)$ Endothelial cell targeting with Tie1-cre. PECAM staining at E13.5 of control $(A)$ and Fgfr1/2 Tie1-cre DCKO $(B)$ hearts demonstrating formation of a normal vascular plexus in Fgfr1/2 Tie1-cre DCKO hearts. ROSA26-LacZ staining of Fgfr1/2 $2^{\text {Tie1-cre }}$ DCKO hearts at E13.5 $(C)$ and E16.5 $(D)$ reveals that Cre-mediated recombination has occurred in endothelial cells. $(E-H)$ Endothelial cell targeting with Flk1-cre. PECAM staining of control $(E)$ and Fgfr1/2 $2^{\text {Flk1-cre }}$ DCKO $(F)$ hearts demonstrating formation of a normal vascular plexus in Fgfr1/2 ${ }^{\text {Flk1-cre }}$ DCKO hearts. ROSA26-LacZ staining of Fgfr1/2 ${ }^{\text {Flk1-cre }}$ DCKO hearts at E13.5 $(G)$ and E16.5 $(H)$ reveals that Cre-mediated recombination has occurred in endothelial cells. Magnification: $A, B, E, F, 25 \times ; C, D, G, H, 400 \times$.

the epicardial marker WT-1 (data not shown). In contrast, at E12.5 formation of the subepicardial mesenchyme was disrupted in Fgfr1/2 ${ }^{\text {MIc2V-cre }}$ DCKO hearts compared with littermate controls (Fig. 4H,K). Further analysis at E13.5 demonstrated that both the subepicardial blood vessels and the vessels growing within the myocardium were impaired in Fgfr1/2 ${ }^{\text {Mlc2v-cre }}$ DCKO hearts (Fig. 4I,L).

Since the vascular plexus of Fofr1/2 $2^{\text {Mlc } 2 v-c r e ~}$ DCKO hearts showed some growth between E11.5 and E13.5, we conclude that loss of FGF signaling to the myocardium imposes defects in coronary development by delaying growth of the vascular plexus. Similar delays were seen in $\mathrm{FgfO}^{-/-}$hearts (Fig. 1; data not shown). Thus, in addition to promoting cardiomyoblast proliferation, FGF signaling controls vascular development, thereby coordinating growth of the coronary vasculature with growth of the myocardium. 


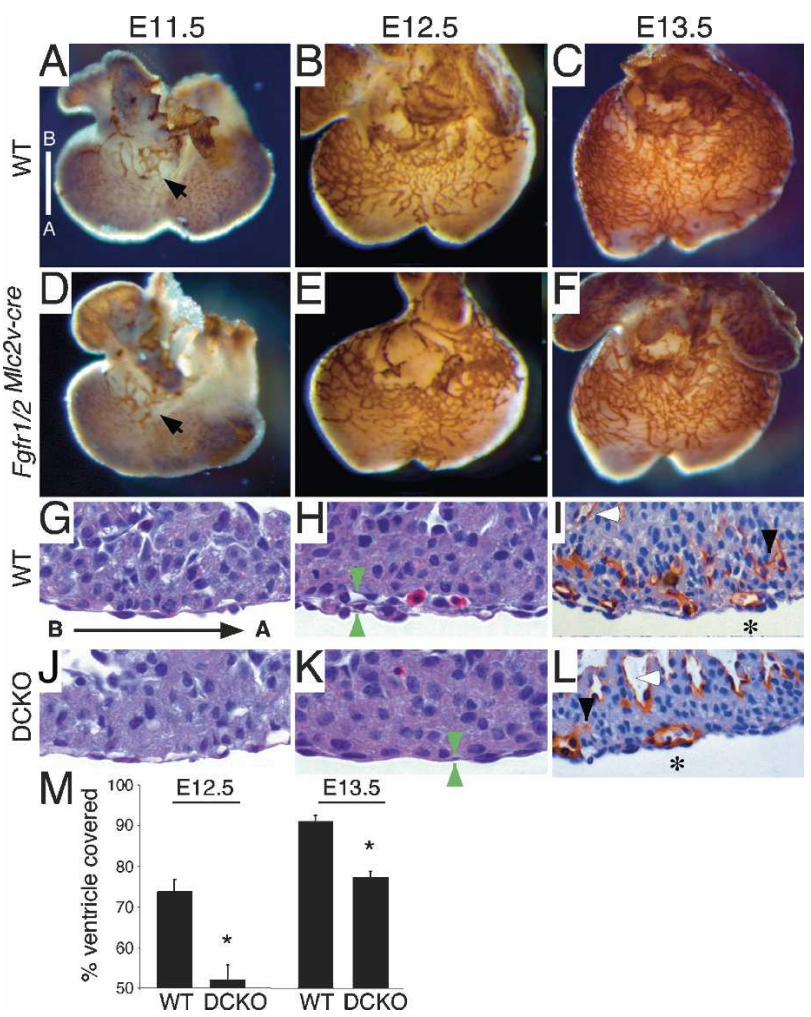

Figure 4. FGF signaling to the cardiomyoblast is essential for coronary vascular development. $(A-F)$ Whole-mount PECAM staining of control $(A-C)$ and Fgfr1/2 ${ }^{\text {Mlc2v-cre }}$ DCKO hearts $(D-$ $F)$. At E11.5, coronary vessels emerge from the atrial-ventricular groove (arrow) of both control $(A)$ and Fgfr1/2 ${ }^{\text {Mlc2v-cre }}$ DCKO $(D)$ hearts. At E12.5 the vascular plexus begins to extend toward the ventricular apices in control hearts $(B)$ but is delayed in Fgfr1/2 ${ }^{\text {Mlc2v-cre }}$ DCKO $(E)$ hearts. By E13.5, control hearts $(C)$ contain a vascular plexus that encases the entire ventricle, while Fgfr1/2 ${ }^{\text {Mlc2v-cre }}$ DCKO hearts $(F)$ contain a vascular plexus that fails to cover the ventricle. $(G-L)$ Histology of control $(G-I)$ and Fgfr $1 / 2^{\text {Mlc2v-cre }}$ DCKO $(J-L)$ hearts demonstrating defects in formation of the subepicardial mesenchyme (green arrowheads) in Fgfr1/2 $2^{\text {Mlc2v-cre }}$ DCKO hearts $(K)$ compared with controls $(H)$ at E12.5. PECAM-stained section of control $(I)$ and Fgfr1/2 ${ }^{\text {Mlc2v-cre }}$ DCKO $(L)$ hearts showing defects in both subepicardial (asterisk) and intramyocardial (black arrowhead) vessel growth at E13.5. The asterisk and black arrowhead mark the position of the most distal subepicardial and intramyocardial blood vessel, respectively. The open arrowhead denotes endocardial PECAM staining. ( $M)$ Quantitation of the percentage of the ventricle covered by blood vessels at E13.5 in control and Fgfr1/2 ${ }^{\text {Mlc2v-cre }}$ DCKO hearts. Asterisk indicates statistically significant differences compared with controls $(p<0.01)$. Magnification: $A-F$, $25 \times ; G-L, 400 \times$. Bar in $A$ and corresponding arrow in $G$ represent orientation and position of histological sections. (B) Base of ventricle; (A) apex of ventricle.

\section{FGF signaling to the cardiomyoblast is necessary for Vegf and Angiopoietin expression}

Based on the above data, FGF signaling promotes coronary development via an indirect mechanism, implying that myocardial FGF signaling must regulate cardiomyoblast-derived signal(s) important for coronary growth. To identify potential cardiomyoblast-derived signals, we took a candidate approach. Vegf-A and Vegf-B have been shown previously to be expressed in the embryonic myocardium during coronary development (Aase et al. 1999; Miquerol et al. 1999; Partanen et al. 1999; Tomanek et al. 2002) and are required for embryonic vascular development (Ferrara et al. 1996; Bellomo et al. 2000; Tammela et al. 2005), and their receptors (Vegfr-1 and Vegfr2) are expressed on vascular endothelial cells (Shalaby et al. 1995; Hiratsuka et al. 1998). In addition, VEGF ligands are regulated by FGF signaling in other cell types (Seghezzi et al. 1998; Kanda et al. 2004). To examine whether Vegf-A, Vegf-B, and other VEGF family members (Vegf-C and Vegf-D) contribute to coronary vascular development, we analyzed the expression of VEGF ligands and their receptors (Vegfr-1, Vegfr-2, and Vegfr-3) in the developing heart at E12.5 and E13.5 using wholemount in situ hybridization.

Vegfr-1 and Vegfr-2 expression was evident in coronary endothelial cells of the developing vascular plexus. Interestingly, cryosections demonstrated expression of Vegfr-1 and Vegfr-2 in both subepicardial and intramyocardial blood vessels (Fig. 5A,B). In contrast, Vegfr-3 was expressed in the epicardium but not in coronary endothelial cells (Fig. 5C). Expression of Vegfr-1 and Vegfr-2 in coronary endothelial cells is consistent with a role for VEGF signaling in coronary development.

Expression analysis of the major VEGF ligands revealed that Vegf-A, Vegf-B, and Vegf-C were expressed in a wave-like pattern during coronary development. At E12.5, Vegf-A, Vegf-B, and Vegf-C were expressed in the atrial-ventricular and interventricular groove (Fig. 5D,G,N). By E13.5, expression of these factors was evident throughout the ventricle (Fig. 5E,H,O). Importantly, the progression of Vegf-A, Vegf- $B$, and Vegf-C expression from E12.5 to E13.5 closely resembled the wave-like growth of the coronary vascular plexus. In contrast, to the other VEGF ligands, Vegf-D was not expressed in a wave-like pattern during coronary development. Instead, Vegf-D expression was restricted to the atrial-ventricular groove and base of the ventricle at both E12.5 and E13.5 (Fig. 5Q,R). Cryosections revealed expression of Vegf-A, Vegf-B, and Vegf-D in the myocardium (Fig. $5 \mathrm{~F}, \mathrm{I}, \mathrm{S})$, while Vegf-C was specifically expressed in cells surrounding the intramyocardial blood vessels (referred to as perivascular cells) (Fig. 5P).

Since Vegf-A, Vegf-B, and Vegf-C expression closely resembled that of the developing coronary plexus at E12.5 and E13.5, these VEGF ligands represented good candidates for the FGF regulated signal critical for coronary development. To determine whether FGF signaling regulates expression of any of these VEGF ligands, control and Fgfr1/2 $2^{\text {Mlc2V-cre }}$ DCKO hearts were subjected to whole-mount in situ hybridization for Vegf-A, Vegf-B, Vegf-C, and Vegf-D. Expression of Vegf-A, Vegf-B, and Vegf-C was both down-regulated and failed to extend from the atrial-ventricular groove toward the ventricular apices in Fgfr1/2 ${ }^{\text {Mlc2v-cre }}$ DCKO hearts (Fig. 5J-M,T,U). Vegf-D expression was unaffected in Fgfr1/2 1 Mlc2v-cre DCKO hearts (Fig. 5V-W). Quantitative RT-PCR (qRT- 
Lavine et al.

Figure 5. FGF signaling to the cardiomyoblast is necessary for Vegf and Angiopoietin expression. $(A-C)$ Whole-mount in situ hybridization for $\operatorname{Vegfr-1}(A)$, Vegfr-2 (B), and Vegfr-3 (C) at E13.5. Vegfr-1 and Vegfr-2 are expressed in coronary endothelial cells, while Vegfr-3 is expressed uniformly within the atrial-ventricular groove. Cryosections reveal Vegfr-1 and Vegfr-2 expression in both subepicardial (asterisk) and intramyocardial (arrowhead) blood vessels and Vegfr-3 expression in epicardial cells (open arrowhead). $(D-F)$ Wave-like progression of $V e g f-A$ expression emanating from the atrial-ventricular and interventricular groove at E12.5 $(D)$ and extending to cover the ventricle by E13.5 $(E)$ in control hearts. $(F)$ Cryosection revealing $V e g f-A$ expression in the myocardium. Open arrowhead denotes lack of staining in the epicardium and subepicardial mesenchyme. $(J, K)$ Vegf- $A$ expression is decreased in Fgfr1/2 ${ }^{\text {Mlc2v-cre }}$ DCKO hearts at E12.5 (J) and E13.5 $(K)$ and fails to cover the ventricle at E13.5 compared with controls (shown in $E$ ). $(G-I)$ Wave-like progression of $V e g f-B$ expression emanating from the atrial-ventricular and interventricular groove at E12.5 $(G)$ and extending to cover the ventricle by E13.5 $(H)$ in control hearts. (I) Cryosection revealing Vegf-B expression in the myocardium. Open arrowhead denotes lack of staining in the epicardium and subepicardial mesenchyme. $(L, M)$ Vegf-B expression is decreased in Fgfr $1 /$ $2^{\text {Mlc2v-cre }}$ DCKO hearts at E12.5 $(L)$ and E13.5 $(M)$ and fails to cover the ventricle at E13.5 compared with controls (shown in $H)$. $(N, O)$ Wave-like progression of $V e g f-C$ expression emanating from the atrial-ventricular and interventricular groove at E12.5 $(N)$ and extending to cover the ventricle by E13.5 $(O)$ in control hearts. $(P)$ Cryosection revealing Vegf-C expression in the perivascular cells (arrowhead). (T,U) Vegf-C expression is decreased in Fgfr1/2 ${ }^{\text {Mlc2v-cre }}$ DCKO hearts at E12.5 (T) and E13.5 $(U)$ and fails to cover the ventricle at E13.5 compared with controls (shown in $O) .(Q, R)$ Vegf-D is expressed in the atrial-ventricular groove at E12.5 (Q) and $\mathrm{E} 13.5(R)$ in control hearts. $(S)$ Cryosections reveal $V e g f-D$ expression in myocardial cells. $(V, W)$ Vegf-D expression is unchanged in Fgfr1/2 ${ }^{\text {Mlc2v-cre }}$ DCKO hearts at both E12.5 $(V)$ and E13.5 $(W) .(X)$ qRT-PCR analysis of control and Fgfr1/2 ${ }^{\text {Mlc2V-cre }}$ DCKO hearts demonstrating decreases in Ang-2, Vegf-A, Vegf-B, and $V e g f-C$ expression. Asterisk represents statistically significant difference compared with controls $(p<0.01)$. All whole-mount specimens were photographed at $25 \times$ magnification and cryosections were photographed at $400 \times$ magnification.

PCR) analysis at E13.5 confirmed statistically significant decreases in Vegf-A, Vegf-B, and Vegf-C mRNA levels in Fgfr1/2 ${ }^{\text {Mlc2v-cre }}$ DCKO hearts (Fig. 5X). Similar decreases in Vegf-A, Vegf-B, and Vegf-C expression were seen in $\mathrm{FgfO}^{-/-}$hearts (Supplementary Fig. 1).

In addition to expressing Vegfr-1 and Vegfr-2, coronary endothelial cells also express Tie2 and the orphan receptor Tie1 (Supplementary Fig. 2). The TIE2 receptor is critical for vascular development and binds Angiopoietin-1 (ANG1) and Angiopoietin-2 (ANG2) (Puri et al. 1999). Engagement by ANG1 is thought to activate TIE2 signaling, while ANG2 antagonizes TIE2 signaling (Ward and Dumont 2002). Previous studies have shown Ang1 and Ang2 expression in the embryonic heart (Partanen et al. 1999; Gale et al. 2002; Ward et al. 2004). To determine whether Ang1 and/or Ang2 are affected by FGF signaling, whole-mount in situ hybridization was performed. Ang1 expression was detected in the atria and atrial-ventricu- lar groove, while Ang2 was expressed in the ventricle at E12.5 (Supplementary Fig. 2). In Fgfr1/2 ${ }^{\text {MIc2v-cre }}$ DCKO hearts, Ang2 expression was found to be significantly down-regulated while Ang1 expression was unaffected (Supplementary Fig. 2). qRT-PCR confirmed these findings (Fig. 5X).

The above analyses indicate that Vegf-A, Vegf-B, Vegf$C$, and Ang2 expression is regulated by FGF signaling to the cardiomyoblast and likely signals to coronary vascular endothelial cells. Thus, these factors represent good candidates for the proposed FGF-regulated cardiomyoblast-derived signal(s) critical for coronary vascular development. Consistent with this possibility Vegf- $A$ and Vegf- $B$ are expressed in cardiomyoblasts; however, Vegf-C is expressed in perivascular cells. Given that myocardial FGF signaling regulates expression of not only Vegf-A and Vegf-B but also Vegf-C, it is likely that FGF signals do so indirectly through an intermediate sig- 
nal. Other support for an intermediate signal includes the following: (1) FGF ligand expression and signaling does not occur in a wave, but rather signals uniformly throughout the ventricle, and (2) FGF function precedes that of VEGF expression by $\sim 2 \mathrm{~d}$, as FGF signaling is required beginning at E10.5 for cardiomyoblast proliferation (Lavine et al. 2005).

\section{FGF signaling to the cardiomyoblast controls a wave} of Hedgehog signaling

Potential intermediate signals include factors that are regulated by FGF signaling and that can promote Vegf and/or Ang2 expression. One candidate that fulfills both criteria is the Hedgehog $(\mathrm{HH})$ signaling pathway. Expression of Sonic hedgehog (Shh), a HH ligand, is positively regulated by mesenchymal FGF signaling in the limb (Lewandoski et al. 2000) and lung (White et al. 2006). In addition, $\mathrm{HH}$ signaling can promote vascular growth, Vegf-A, and Ang2 expression, both in vitro and in vivo, and is necessary for early embryonic vascular development (Pola et al. 2001; Kanda et al. 2003; Vokes et al. 2004).

To determine whether $\mathrm{HH}$ signaling is active in the embryonic heart and could constitute this intermediate signal, we examined the expression of $\mathrm{HH}$ ligands (Shh, $D h h$, and $I h h$ ) and their receptor Ptc1 during coronary development. In situ hybridization for Shh, Ihh, and Dhh revealed that $S h$ was the primary $\mathrm{HH}$ ligand expressed during coronary development, as only low levels of $\mathrm{Ih}$ and Dhh could be detected (Fig. 6A,B; data not shown). Shh was expressed in the atrial-ventricular groove and base of the ventricle at E12.5 and E13.5. Cryosections showed Shh expression in the epicardium (Fig. 6F). Immunohistochemical analysis confirmed epicardial expression of SHH protein (Fig. 6G).

Ptc1 is directly up-regulated by $\mathrm{HH}$ signaling via downstream GLI transcription factors and thus can be used as a reporter of $\mathrm{HH}$ signaling (Pearse et al. 2001). Examination of Ptc1 expression during coronary development revealed that Ptc1 was expressed in the atrialventricular and interventricular groove at E12.5 (Fig. $6 \mathrm{H})$. By E13.5, Ptc1 expression was present throughout the ventricle (Fig. 6I). Cryosections and immunohistochemical analysis showed Ptc1 expression in both cardiomyoblasts and perivascular cells (Fig. 6L-O). Importantly, similar to Vegf-A, Vegf-B, and Vegf-C, Ptc1 expression closely resembled the wave-like progression of the coronary vascular plexus as it migrated over the heart between E12.5 and E13.5. These data indicate that $\mathrm{HH}$ signaling travels down the heart coincident with both the wave-like expansion of Vegf expression and growth of the coronary vascular plexus.

If $\mathrm{SHH}$ is the intermediate signal between FGF and VEGF signaling, then we would expect that Shh expression and $\mathrm{HH}$ signaling would be diminished in Fgfr1/ $2^{\text {Mlc2v-cre }}$ DCKO hearts. Examination of Shh expression by whole-mount in situ hybridization and qRT-PCR demonstrated a near absence of Shh expression in Fgfr1/ $2^{\text {Mlc2v-cre }}$ DCKO hearts at E12.5 (Fig. 6C,E). Analysis of Ptc1 expression revealed both diminished expression and failure of Ptc1 expression to spread from the interventricular and atrial-ventricular grooves at E12.5 toward the ventricular apices by E13.5 (Fig. 6H-K). Similar defects in Shh and Ptc1 expression were present in $\mathrm{FgfO}^{-/-}$ hearts (Supplementary Fig. 3).
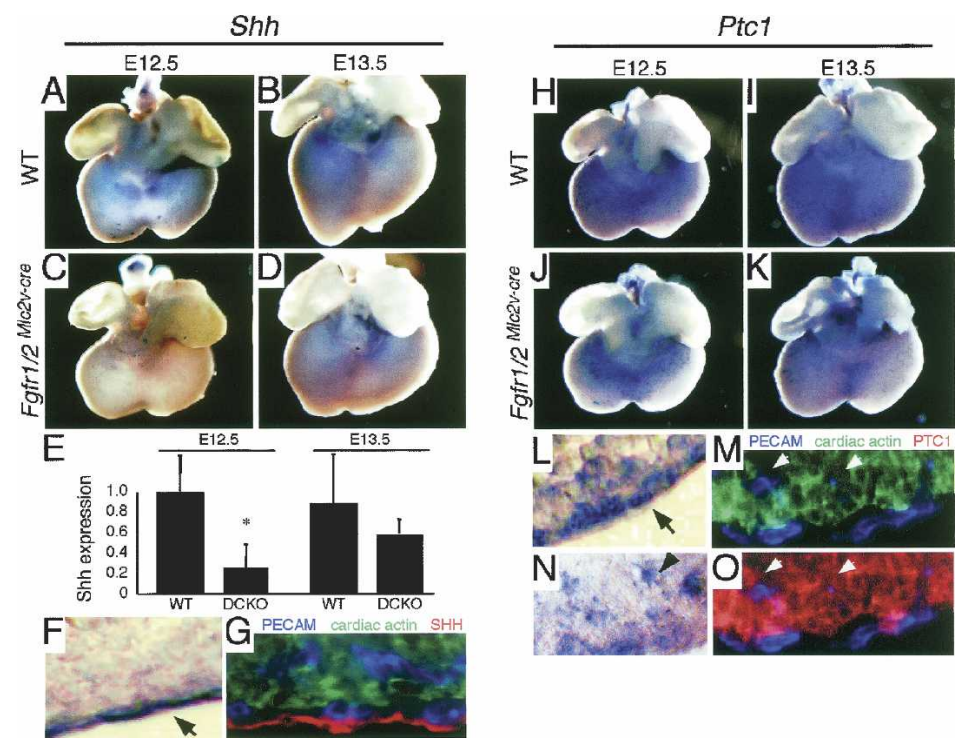

Figure 6. FGF signaling to the cardiomyoblast controls a wave of $\mathrm{HH}$ signaling. $(A-F)$ Whole-mount in situ hybridization for $S h h$. Shh is expressed in the atrial-ventricular groove at E12.5 $(A)$ and E13.5 (B). At E12.5 (C) Shh expression is nearly absent from Fgfr $1 / 2^{\text {Mlc2v-cre }}$ DCKO hearts but begins to be expressed by E13.5 (D). (E) qRT-PCR demonstrating Shh mRNA decrease in Fofr $1 / 2^{\text {Mlc2v-cre }}$ DCKO hearts $(p<0.01)$. (F) Cryosections of $A$ reveal Shh expression in the epicardium. (G) Immunohistochemistry showing SHH protein expression in the epicardium (red). (Blue) PECAM; (green) cardiac actin. $(H-O)$ Whole-mount in situ hybridization for Ptc1 demonstrating a wave-like progression of Ptc1 expression between E12.5 and E13.5. Ptc1 is expressed in the atrial-ventricular and interventricular groove at E12.5 $(H)$ and extends to cover the ventricle by E13.5 $(I)$. Ptc1 expression is decreased compared with controls at both E12.5 $(J)$ and E13.5 $(K)$ in Fgfr1/2M1c2v-cre DCKO hearts. Ptc1 expression also fails to progress in a wavelike fashion and does not cover the ventricle at E13.5 in Fgfr1/2 ${ }^{\text {M1c2v-cre }}$ DCKO hearts. Cryosections of $H$ reveal Ptc1 expression in the cardiomyoblasts $(L$, arrow) and perivascular cells $(N$, arrowhead). Immunohistochemistry showing PTC1 protein expression (red) in the myocardium and perivascular cells. $(M)$ Cryosection showing cardiac actin and PECAM expression. (O) Same section as $M$, but showing PTC1 and PECAM expression. (Blue) PECAM; (green) cardiac actin. White arrowheads indicate perivascular cells positive for PTC1 (O) but lacking cardiac actin staining $(M)$. All whole-mount specimens were photographed at $25 \times$ magnification and cryosections were photographed at $400 \times$ magnification. 
Lavine et al.

Interestingly, Shh expression could be detected at E13.5 in Fgfr1/2 $2^{\text {Mlc2v-cre }}$ DCKO hearts (Fig. 6D,E). The delay in Shh expression seen in Fgfr1/2 $2^{\text {Mlc2v-cre }}$ DCKO hearts may explain why growth of the vascular plexus is similarly delayed and not absent in Fgfr1/2Mlc2v-cre DCKO hearts.

\section{FGF signaling induces Vegf expression in a HH-dependent manner}

The second criteria that SHH must meet to be the intermediate signal between FGF and VEGF is that SHH must regulate Vegf- $A$, Vegf-B, and Vegf-C expression. To determine whether $\mathrm{SHH}$ fulfills this role, we utilized a heart slice organ culture system similar to that previously described (Stuckmann et al. 2003). To test the feasibility of this system, we treated heart slices with FGF9 protein for $24 \mathrm{~h}$ and examined Vegf-A, Vegf-B, and Vegf-C expression by either LacZ staining (VEGFA-LacZ) (Miquerol et al. 1999) or whole-mount in situ hybridization. As expected, FGF9 treatment led to induction of $V e g f-A, V e g f-B$, and Vegf-C expression compared with the BSA control (Fig. 7A,B; Supplementary Fig. $4 \mathrm{~A}, \mathrm{~B}, \mathrm{G}, \mathrm{H})$. To test whether SHH could lead to similar increases in $V e g f$ ligand expression, we examine expression of Vegf-A, Vegf-B, and Vegf-C after treatment with
$\mathrm{SHH}$ protein. $\mathrm{SHH}$ treatment also resulted in marked induction of $V e g f-A, V e g f-B$, and $V e g f-C$ expression (Fig. 7C; Supplementary Fig. 4C-I).

The above data indicate that both FGF and HH signaling can promote VEGF ligand expression. Since FGF signaling is necessary for Shh expression in the heart, we hypothesized that FGF9 may regulate Vegf ligand expression by activating $\mathrm{HH}$ signaling. Note that this would implicate $\mathrm{SHH}$ as an intermediate signal between FGF and VEGF. To test whether HH signaling is necessary for FGF9 to induce Vegf ligand expression, we treated heart slices with either BSA, FGF9, or SHH protein in the presence of the $\mathrm{HH}$ antagonist cyclopamine. Consistent with the above hypothesis, application of cyclopamine abolished the ability of both FGF9 and SHH protein to induce $V e g f-A, V e g f-B$, and Vegf-C expression, indicating that $\mathrm{HH}$ signaling is necessary for FGF9 to promote Vegf ligand expression (Fig. 7D-F; Supplementary Fig. 4D-F,JL). Control experiments demonstrated that cyclopamine was able to inhibit $\mathrm{HH}$ signaling (Ptc1 expression) and did not lead to lethality of the explant (presence of beating and $\beta$-Myosin Heavy Chain [Mhc] expression) (Supplementary Fig. 4M-X).

To determine whether $\mathrm{HH}$ signaling is sufficient to induce Vegf ligand expression, Fgfr1/2 $2^{\text {Mlc2c-cre }}$ DCKO explants were treated with either BSA, FGF9, or SHH pro-
Figure 7. FGF-HH pathway regulates coronary vascular development and VEGF expression. $(A-C)$ Treatment of heart slices with either FGF9 or SHH led to induction of Vegf-A expression compared with BSA controls. $(D-F)$ Addition of the $\mathrm{HH}$ inhibitor, cyclopamine, ablated the ability of either FGF9 or SHH protein to induce Vegf-A expression compared with BSA controls. Vegf-A expression was detected by LacZ staining for a VEGFA-LacZ genetrap. $(G-L)$ Treatment of control $(G-I)$ or Fgfr1/2 ${ }^{\text {Mlc2v-cre }}$ DCKO $(J-L)$ heart slices with BSA, FGF9 and SHH protein. In response to FGF9 protein, control hearts $(H)$ showed robust increases in $\operatorname{Vegf-A}$ expression, while Fgfr1/2 ${ }^{\text {Mlc2v-cre }}$ DCKO $(K)$ hearts showed no induction of Vegf- $A$ expression compared with BSA control. In contrast, SHH treatment of both control $(I)$ and Fgfr1/2 ${ }^{\text {Mlc2v-cre }}$ DCKO $(L)$ hearts led to robust increases in Vegf-A expression. $V e g f-A$ expression was assayed by in situ hybridization. $(M-P)$ PECAM staining of whole hearts cultured for $24(M)$ or $48(N)$ $\mathrm{h}$, demonstrating that coronary vessels grow in a wave-like fashion. $(\mathrm{O}-\mathrm{P})$ Cyclopamine treatment abolishes coronary vessel development. (Q-T) In situ hybridization showing that cyclopamine treatment suppresses Vegf-A expression at both $24 \mathrm{~h}(S)$ and $48 \mathrm{~h}(T)$ compared with controls $(Q, R)$.

$(U-Y)$ PECAM staining indicating that coronary defects due to cyclopamine treatment $(V)$ can be rescued by cotreatment with

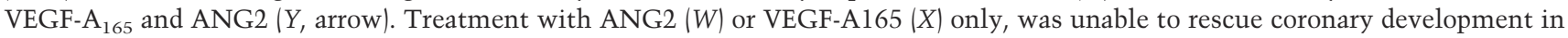
cyclopamine-treated hearts. Hearts were cultured for $48 \mathrm{~h}$. All explants were photographed at $32 \times$ magnification.
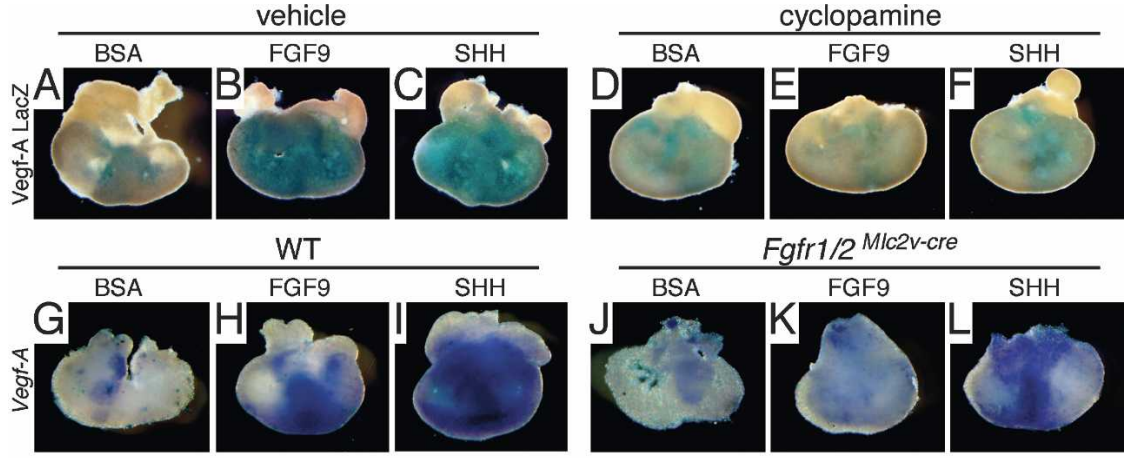

PECAM

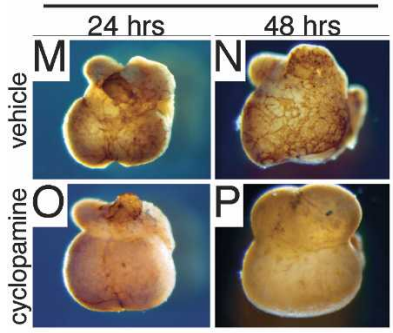

Vegf- $A$
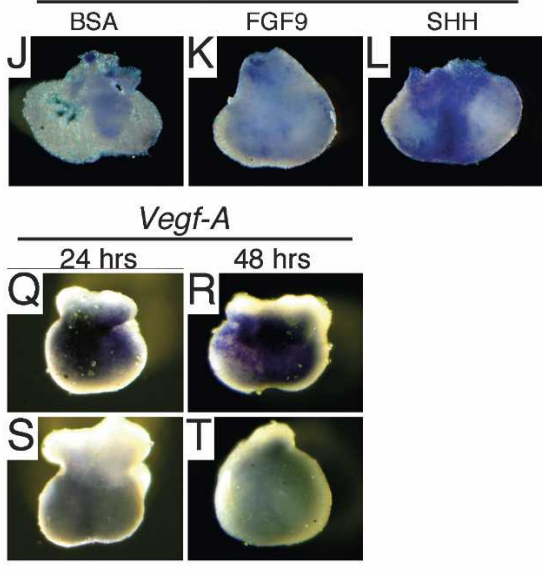

cyclopamine cyclopamine
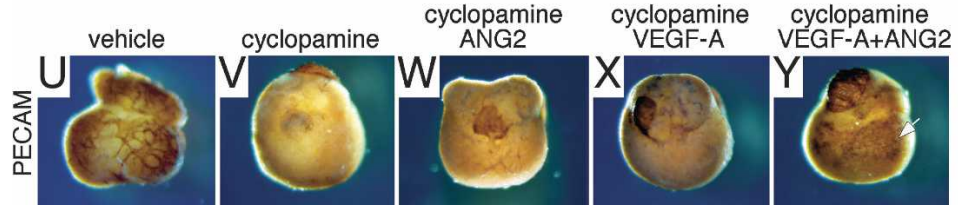
tein and assayed for Vegf-A expression. Treatment of control heart slices with either FGF9 or SHH protein led to a robust increase in $V e g f-A$ expression compared with BSA controls (Fig. 7G-I). In contrast, only treatment of Fgfr1/2 $2^{\text {Mlc2c-cre }}$ DCKO heart slices with $\mathrm{SHH}$, but not FGF9, resulted in induction of Vegf-A expression (Fig. 7J-L). This indicates that (1) FGF9 controls Vegf- $A$ expression by signaling to the cardiomyoblast, and (2) $\mathrm{SHH}$ signaling is sufficient to promote $V e g f-A$ expression in the absence of myocardial FGF signaling. Together these experiments place $\mathrm{SHH}$ downstream of FGF signaling and strongly implicate $\mathrm{SHH}$ as the intermediate signal between FGF and VEGF.

\section{HH signaling is essential for coronary development}

Because the slice cultures do not allow visualization of the developing coronary vasculature, we utilized a second organ culture system to confirm that $\mathrm{HH}$ signaling is necessary for VEGF ligand expression and to determine whether $\mathrm{HH}$ signaling is essential for coronary development. For these cultures, E11.5 whole hearts were dissected and cultured in the presence or absence of cyclopamine for 24-48 h with gentle rocking.

PECAM staining of hearts cultured with vehicle only revealed that the coronary vascular plexus formed in an almost identical manner to in vivo. At $24 \mathrm{~h}$, blood vessels were present in the atrial-ventricular and interventricular grooves. By $48 \mathrm{~h}$, the vascular plexus expanded to cover the entire ventricle, consistent with the wave-like pattern of growth that occurs in vivo (Fig. $7 \mathrm{M}, \mathrm{N})$. Treatment of hearts with $10 \mu \mathrm{M}$ cyclopamine completely disrupted coronary vascular growth, as no blood vessels could be detected at either 24 or $48 \mathrm{~h}$ of culture (Fig. $7 \mathrm{O}, \mathrm{P})$. This indicates that $\mathrm{HH}$ signaling is required for coronary vascular development.

In situ hybridization of cyclopamine-treated hearts demonstrated that, in addition to defects in coronary vessel growth, Vegf-A expression was suppressed (Fig. 7Q-T). qRT-PCR experiments confirmed statistically significant reduction in Vegf- $A$ expression, and additionally, revealed diminished Vegf-B, Vegf-C, and Ang2 expression. Vegf-D and Ang1 were unaffected (data not shown). These data demonstrate that $\mathrm{HH}$ signaling is necessary for coronary vascular development, Vegf- $A$, Vegf-B, Vegf-C, and Ang2 expression.

To determine whether $\mathrm{HH}$ signaling controls coronary vascular growth by inducing VEGF and Ang2 expression, we treated hearts cultured in the presence of cyclopamine with either VEGF-A ${ }_{165}$, ANG2, or both factors. As expected, cyclopamine treated hearts failed to form a vascular plexus at $48 \mathrm{~h}$ (Fig. 7U,V). Addition of ANG2 or VEGF-A 165 alone was unable to rescue coronary defects in cyclopamine-treated hearts (Fig. $7 \mathrm{~W}, \mathrm{X}$ ). In contrast, simultaneous treatment with ANG2 and VEGF-A 165 rescued development of the coronary vascular plexus (Fig. 7Y). These data indicate that (1) Ang2 and VEGF ligands are the relevant targets of $\mathrm{HH}$ signaling during coronary development, and (2) ANG2 and VEGF act synergistically to promote vascular plexus formation.
Activation of $\mathrm{HH}$ signaling rescues coronary defects in hearts lacking myocardial FGF signaling

Explant culture studies demonstrated that $\mathrm{SHH}$ functions downstream of FGF signaling and is sufficient to promote coronary development in the absence of FGF function. These findings suggested that FGF signaling may regulate coronary development solely by controlling Shh expression. That is, the only contribution FGF signaling makes toward regulating coronary development is through up-regulation of $\mathrm{SHH}$.

To test this hypothesis, we asked whether activation of $\mathrm{HH}$ signaling could rescue the coronary defects seen in Fgfr1/2 ${ }^{\text {Mlc2v-cre }}$ DCKO hearts in vivo. To promote $\mathrm{HH}$ activation in vivo, we utilized a transgenic line (pCAGGs$\mathrm{LacZ}^{\text {flox}}$-GLI2 ${ }^{\star}$, referred to as GLI2 ${ }^{\star}$ ) that expresses an activated form of GLI2 upon CRE-mediated recombination (Mill et al. 2003). In addition to expression of activated GLI2, CRE-mediated recombination removes a loxp flanked LacZ cassette to allow monitoring of recombination efficiency by loss of LacZ expression (Fig. 8A,B).

To assay whether activation of the GLI2* transgene can lead to forced activation of $\mathrm{HH}$ signaling in the heart, we crossed GLI2* with Mlc2v-Cre mice (Fig. 8A,B). PECAM staining of GLI2*/Mlc2v-Cre hearts revealed a normally patterned vascular plexus at E13.5 (Fig. 8C,D). High magnification of the vasculature in GLI2*/Mlc2vCre hearts demonstrated an increased density of blood vessels (Fig. 8E,F). Histological sections of PECAMstained hearts showed an increase in the number of subepicardial blood vessels in GLI2*/Mlc2v-cre hearts compared with controls, $12.2 \pm 1.0$ and $9.8 \pm 0.8$ vessels $/ 20 \times$ field, respectively $(p<0.0001)$ (Fig. $8 \mathrm{G}-\mathrm{I})$. There was no statistically significant change in the number of intramyocardial blood vessels in GLI2*/Mlc2v-Cre hearts compared with controls, $15.6 \pm 1.5$ and $14.2 \pm 1.2$ vessels $/ 20 \times$ field, respectively $(p=0.055)$. Whole-mount in situ hybridization of GLI2*/Mlc2v-Cre hearts at E12.5 demonstrated up-regulation of Ptc1 and Vegf-A, consistent with activation of $\mathrm{HH}$ signaling (Fig. 7J-M). Increased Ptc1 and Vegf-A expression in GLI2*/Mlc2v-Cre hearts was confirmed by qRT-PCR. Elevations in Ang-2 expression were also detected (Fig. 8N).

To test the hypothesis that activation of $\mathrm{HH}$ signaling is the sole function of FGF signaling in coronary development, we bred the GLI2* transgene into the Fgfr1/ $2^{\text {Mlc2v-cre }}$ DCKO line to generate mice of the genotype Fgfr1/2 ${ }^{\text {Mlc2v-cre }}$;GLI2* ${ }^{*}$ In these mice, cardiac specific CRE-recombination leads to concurrent inactivation of both Fgfr1 and Fgfr2 and activation of $\mathrm{HH}$ signaling in the cardiomyoblast.

PECAM staining of control, Fgfr1/2 ${ }^{\text {Mlc2v-cre }}$, and Fgfr1/ $2^{\text {Mlc2v-cre }}$;GLI2 * hearts at E13.5 demonstrated the presence of a vascular plexus that encased the ventricle of control hearts, and as expected, Fgfr1/2 $2^{\text {Mlc2v-cre }}$ DCKO hearts displayed defects in vascular plexus growth (Fig. $9 \mathrm{~A}, \mathrm{~B})$. Consistent with a rescue, Fgfr1/2 ${ }^{\text {Mlc2v-cre }}$;GLI2 * hearts contained a normal-appearing vascular plexus that covered the entire ventricle (Fig. 9C). Quantitation of the percentage of the ventricle covered by blood ves- 
Lavine et al.

Figure 8. Activation of $\mathrm{HH}$ signaling in the embryonic myocardium increases subepicardial blood vessel growth. $(A, B)$ LacZ staining of control $(A)$ and GLI2*/Mlc2v-Cre $(B)$ hearts at E12.5. Reduction of LacZ staining in GLI2*/Mlc2v-Cre hearts demonstrates efficient Cre-mediated recombination of the transgene throughout the ventricular myocardium. $(C-F)$ PECAM staining of control $(C, E)$ and GLI2*/Mlc2v-Cre $(D, F)$ hearts. Both control $(C)$ and GLI2*/Mlc2v-Cre $(D)$ hearts contain a normally patterned coronary plexus at E13.5. High magnification reveals that GLI2*/Mlc2v-Cre hearts $(F)$ have a denser coronary plexus than controls $(E)$. $(G, H)$ Histological sections of PECAM-stained control $(G)$ and GLI2*/Mlc2v-Cre $(H)$ hearts reveals increased number of subepicardial blood vessels. (I) Quantitation of blood vessel number/20x field demonstrated statistically significant increases in subepicardial but not intramyocardial blood vessels $(p<0.01) .(J, K)$ In situ hybridization for Ptc1 expression showing increased expression in GLI2*/ Mlc2v-Cre hearts $(K)$ compared with controls $(J)$. $(L, M)$ In situ hybridization for Vegf- $A$ expression showing increased expression in GLI2*/Mlc2v-Cre hearts $(M)$ compared with controls $(L) .(N)$ qRTPCR analysis of control and GLI2*/Mlc2 v-Cre hearts revealing significant increases in Ang2, Ptc1, and Vegf-A expression. Asterisk indicates statistically significant differences compared with controls $(p<0.01)$. All whole-mount specimens except $E$ and $F$ were photographed at $25 x ; E$ and $F$ were photographed at $90 \times$ magnification. Histological sections were photographed at 400× magnification.

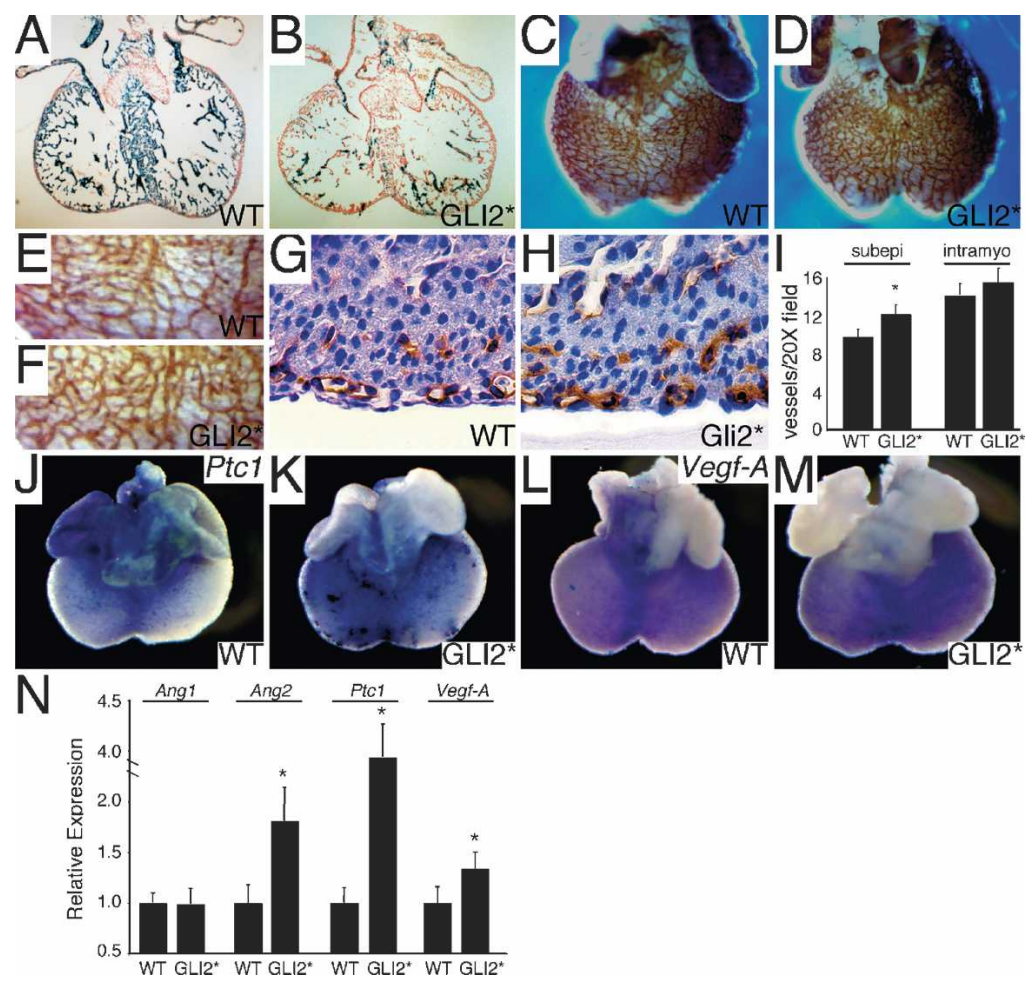

sels revealed that control and Fgfr1/2 ${ }^{\text {Mlc2v-cre }}$ DCKO hearts had $90 \pm 1 \%$ and $79 \pm 2 \%$ of the ventricle covered by blood vessels, respectively $(p<0.001)$. Fgfr1/2 $2^{\text {Mlc2v-cre }}$; GLI2* hearts had $93 \pm 0.2 \%$ of the ventricle covered by blood vessels, a nonsignificant difference compared with controls $(p=0.18)$ but statistically significant difference compared with Fgfr1/2 $2^{\text {Mlc2v-cre }}$ DCKO hearts $(p<0.001)$ (Fig. 9J).

To further address whether activation of $\mathrm{HH}$ signaling in Fgfr1/2 ${ }^{\text {Mlc2v-cre }}$ DCKO hearts could rescue defects in coronary development, Fgfr1/2 $2^{\text {Mlc2v-cre }}$ DCKO and Fgfr1/ $2^{\text {MIc2V-cre }}$;GLI2 * hearts were subjected to whole-mount in situ hybridization for Vegf-A. Consistent with the ability of GLI2 ${ }^{\star}$ to rescue the coronary defects of Fgfr1/ $2^{\text {Mlc2V-cre }}$ DCKO hearts, activation of $\mathrm{HH}$ signaling in Fgfr1/2 ${ }^{\text {Mlc2v-cre }}$ DCKO hearts restored Vegf-A expression to grossly normal levels (Fig. 9D-F,K).

Interestingly, histological analysis of PECAM-stained control, Fgfr1/2 ${ }^{\text {Mlc2v-cre }}$ DCKO, and Fgfr1/2 ${ }^{\text {Mlc2v-cre }}$; GLI2* hearts revealed that only subepicardial blood vessel growth was rescued in Fgfr1/2 ${ }^{\text {Mlc2v-cre }}$;GLI2* hearts (Fig. 9G-I). This finding is consistent with elevations in subepicardial but not intramyocardial blood vessel number in GLI2*/Mlc2v-Cre hearts (Fig. 8G,H), suggesting that activation of $\mathrm{HH}$ signaling in the cardiomyoblast specifically controls subepicardial blood vessel growth. These data suggest that SHH signals to the cardiomyoblast to control subepicardial blood vessel growth and may signal to another cell type (such as perivascular cells) to control intramyocardial blood vessel growth. Perivascular cells express Ptc1 and Vegf-C in a HH-dependent manner, consistent with a role for these cells in intramyocardial blood vessel development.

\section{Activation of HH signaling in the adult heart increases coronary vessel density}

Collectively, the above experiments uncover an essential role for $\mathrm{HH}$ signaling in the development of the coronary vascular system and further demonstrate that activation of $\mathrm{HH}$ signaling can promote coronary vessel growth in the embryonic heart. To examine whether activation of $\mathrm{HH}$ signaling can promote coronary growth in the adult heart, we crossed GLI2* and $\alpha$ MHC-ER-Cre mice (Sohal et al. 2001) to generate GLI2* $/ \alpha$ MHC-ERCre animals. $\alpha$ MHC-ER-Cre mice express cytoplasmicrestricted CRE recombinase specifically in cardiomyocytes. Injection of tamoxifen leads to nuclear translocation of CRE and subsequent CRE-mediated recombination. Thus, by injecting tamoxifen into GLI2*/ $/ \alpha$ MHC-ER-Cre animals, we can induce $\mathrm{HH}$ signaling in the adult myocardium.

To activate $\mathrm{HH}$ signaling in the adult heart, we injected GLI2*/ $\alpha$ MHC-ER-Cre animals with tamoxifen once per day for three consecutive days. Three to five consecutive injections of tamoxifen leads to efficient re- 


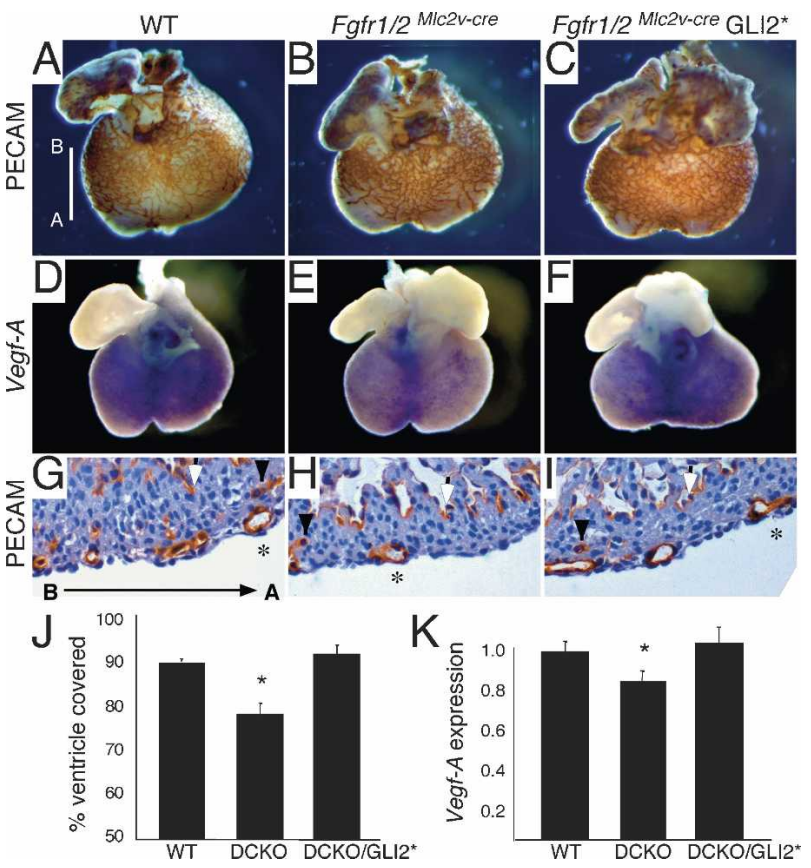

Figure 9. Activation of $\mathrm{HH}$ signaling rescues coronary defects in hearts lacking myocardial FGF signaling. $(A-C)$ PECAM staining of control $(A), F g f r 1 / 2^{\text {Mlc2v-cre }}$ DCKO $(B)$, and Fgfr1/ $2^{\text {MIc2v-cre }}$ GLI2* hearts $(C)$ at E13.5. Control hearts contained a vascular plexus that encased the entire ventricle, while Fgfr1/ $2^{\text {Mlc2v-cre }}$ DCKO hearts contained a vascular plexus that failed to enclose the ventricle. Fgfr1/2 ${ }^{\mathrm{MIc} 2 \mathrm{v}-\mathrm{cre}}$; GLI2 ${ }^{\star}$ hearts contained a vascular plexus that, like controls, covered the ventricle. $(D-F)$ In situ hybridization for Vegf- $A$ in control $(D)$, Fgfr1/2 $2^{\text {Mlc2v-cre }}$ DCKO $(E)$, and Fgfr1/2 ${ }^{\text {Mlc2v-cre }} ;$ GLI2 ${ }^{\star}$ hearts $(F)$. Fgfr1/2 ${ }^{\text {Mlc2v-cre }}$ DCKO hearts displayed decreased and restricted Vegf-A expression, while Fgfr1/2 ${ }^{\text {MIc2v-cre }}$;GLI2* hearts displayed Vegf-A expression indistinguishable from controls. $(G-I)$ Histological sections of E13.5 PECAM-stained hearts. Fgfr1/2 1 Mlc2v-cre DCKO hearts $(H)$ had decreased subepicardial (asterisk) and intramyocardial (black arrowhead) blood vessels compared with controls (G). (I) Fgfr1/2 ${ }^{\text {Mlc2v-cre }}$;Gli2* hearts displayed rescued subepicardial (asterisk) but not intramyocardial (black arrowhead) blood vessel development. The asterisk and black arrowhead mark the position of the most distal subepicardial and intramyocardial blood vessel, respectively. Open arrowhead denotes endocardial PECAM staining. (J) Quantitation of the percent of the ventricle covered by blood vessels at E13.5 demonstrating that only Fgfr1/ $2^{\text {Mlc2v-cre }}$ DCKO hearts had statistically significant differences from controls. Asterisk indicates a statistically significant difference $(p<0.01)$. (K) qRT-PCR analysis confirming that Fgfr 1/ $2^{\text {Mlc2v-cre }} ;$ GLI2 ${ }^{*}$ hearts contain wild-type levels of Vegf-A. Asterisk indicates a statistically significant difference $(p<0.01)$ compared with both control and Fgfr1/2 ${ }^{\text {Mlc2v-cre }}$;GLI2 ${ }^{\star}$ hearts. Bar in $A$ and corresponding arrow in $G$ represent orientation and position of histological sections. (B) Base of ventricle; (A) apex of ventricle. All whole-mount specimens were photographed at $25 \times$. Histological sections were photographed at $400 \times$ magnification.

combination in the adult myocardium (Sohal et al. 2001). Mice were sacrificed on day 5 and hearts were harvested.

Histological examination revealed hypercellularity in the interstitial space between individual cardiomyocytes in GLI2*/ $\alpha$ MHC-ER-Cre hearts compared with controls
(Fig. 10A,B). Examination of coronary vessel density by PECAM staining demonstrated an increase in blood vessel number, specifically small vessels located within the interstitium (Fig. 10C,D). To determine whether these HH-induced blood vessels are connected to the coronary vascular system, we injected biotinylated Tomato Lectin into the inferior vena cava of control and GLI2* $/ \alpha$ MHCER-Cre animals. Streptavidin-HRP staining demonstrated increased Lectin-positive vessels in GLI2*/ $\alpha$ MHC-ER-Cre compared with control hearts, indicating that the $\mathrm{HH}$-induced vasculature is contiguous with the systemic circulation (Fig. 10E,F). To quantitate increases in blood vessel number, we performed qRT-PCR analysis for Pecam and Vegfr-2. Pecam mRNA content was increased 1.4-fold $(p<0.001)$ and Vegfr-2 content was increased 1.8 -fold $(p<0.001)$ in GLI2* $/ \alpha$ MHC-ER-Cre compared with control hearts (Fig. 10G).

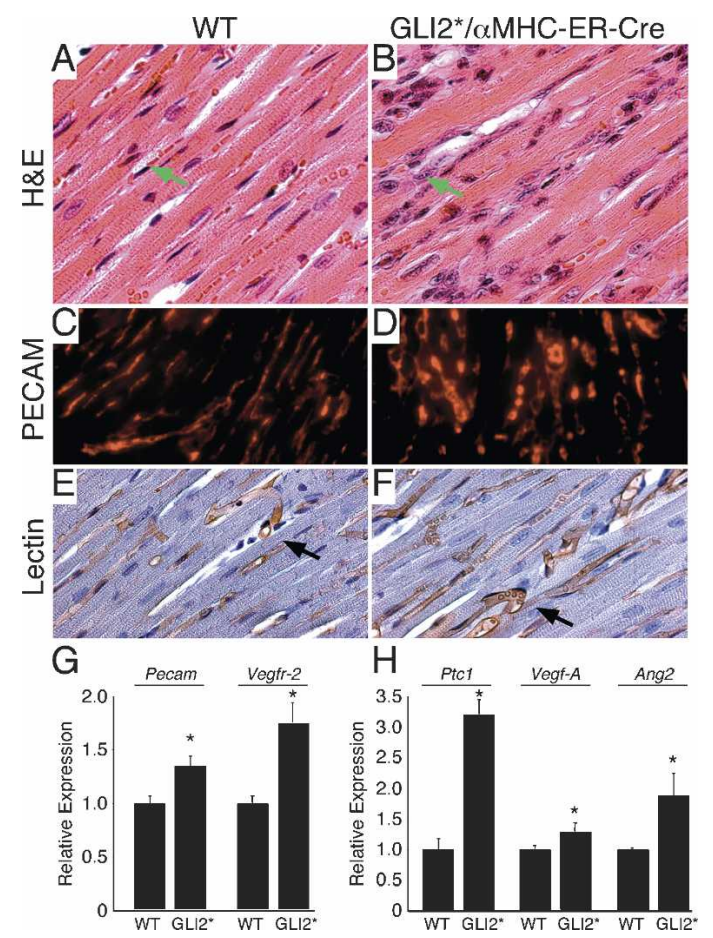

Figure 10. Activation of $\mathrm{HH}$ signaling in the adult heart increases coronary vessel density. $(A, B)$ Histological sections of control $(A)$ and GLI2* $* \alpha M H C$-ER-Cre $(B)$ hearts after $5 \mathrm{~d}$ of transgene induction showing hypercellularity in the interstitial space (green arrow). $(C, D)$ Immunofluorescent PECAM staining of control $(C)$ and GLI2*/ $/ \alpha$ HC-ER-Cre $(D)$ hearts revealing increased coronary vessel density in the interstitial space. $(E, F)$ Streptavidin-HRP staining (arrows) following intravascular injection of biotinylated Tomato Lectin into control $(E)$ and GLI2*/ ${ }^{\star}$ MHC-ER-Cre $(F)$ animals demonstrates that HH-induced blood vessels are connected to the systemic vasculature. $(G)$ Quantitation of increased coronary vessel number by qRTPCR demonstrating that GLI2*/ $\alpha$ MHC-ER-Cre hearts have significantly elevated levels of Pecam and Vegfr-2 expression. $(H)$ qRT-PCR indicating increased Ang2, Ptc1, and Vegf-A expression in GLI2*/ $\alpha$ MHC-ER-Cre hearts. Asterisk indicates statistically significant differences compared with controls $(p<0.01)$. Histological sections were photographed at $400 \times$ magnification. 
Based on our embryonic analyses, we expected that $\mathrm{HH}$ signaling promoted coronary growth by increasing Vegf and Ang2 expression. Consistent with this prediction, qRT-PCR analysis demonstrated statistically significant increases in Ptc1, Vegf-A, and Ang2 mRNA content (Fig. 10H). Ang1 expression was unaltered (data not shown).

The above study demonstrates that activation of $\mathrm{HH}$ signaling in the cardiomyocyte leads to increased coronary vessel number in the adult heart after only $5 \mathrm{~d}$ of transgene activation. The ability of $\mathrm{HH}$ signaling to induce significant increases in vascular density in a relatively short time suggests that $\mathrm{HH}$ signaling is a potentially important target for therapy aimed at increasing coronary vascularization and perfusion in the context of myocardial ischemia and acute myocardial infarction.

\section{Discussion}

HH signaling is a novel therapeutic target for coronary neoangiogenesis

Despite the widespread success of FGF2- and VEGF$\mathrm{A}_{165}$-mediated induction of coronary neovascularization in animal models, these therapies have produced disappointing results in recent phase 2 clinical trials (Syed et al. 2004). By dissecting the molecular mechanisms that govern formation of the coronary vascular system during development, we have identified a new pharmacological target for promoting coronary neovascularization. Activation of $\mathrm{HH}$ signaling is both critical for coronary development and sufficient to promote formation of new coronary vessels in the embryonic and adult heart. Our work is consistent with results recently published demonstrating that Shh gene therapy can promote coronary neovascularization and protect from ischemic injury in rodent and large animal models (Kusano et al. 2005). Collectively, these data make a compelling case for the potential therapeutic use of $\mathrm{HH}$ agonists in patients with ischemic heart disease.

$\mathrm{HH}$ signaling orchestrates coronary development by controlling the expression of multiple proangiogenic factors, including Vegf-A, Vegf-B, Vegf-C, and Ang2. Given that $\mathrm{HH}$ signaling regulates coronary vascular formation, promotes neovascularization in the adult heart, and induces expression of numerous signaling molecules, we suggest that $\mathrm{HH}$ signaling constitutes an essential regulator of coronary vessel growth.

Therapeutics aimed at such a critical regulator of coronary growth have the potential to succeed where other pharmacological modalities have failed. It has been postulated that monotherapy with FGF2 or VEGF-A ${ }_{165}$ has been unsuccessful because expression of multiple factors are required for efficient coronary neovascularization (Syed et al. 2004). Consistent with this concept, coexpression of $V e g f-A$ and $A n g 2$ in the myocardium leads to significantly more robust increases in coronary density than Vegf-A or Ang2 alone (Visconti et al. 2002). Intriguingly, $\mathrm{HH}$ signaling regulates expression of not only $V e g f-A$ and $A n g 2$ but also Vegf-B and Vegf-C, making $\mathrm{HH}$ signaling an attractive candidate for therapy aimed at promoting coronary vascular growth.

The epicardium acts as a signaling center for heart development

Removal of the epicardium leads to defects in cardiomyoblast proliferation and coronary development (Morabito et al. 2002; Reese et al. 2002; Wada et al. 2003). Previously, we have shown that epicardial-derived FGF signals are essential mediators of myocardial growth (Lavine et al. 2005). In addition to this mitogenic activity, we show here that epicardial- and endocardial-derived FGF signals also control coronary vascular development. These findings demonstrate that the epicardium acts as a source of signaling molecules critical for heart development.

The overwhelming majority of mutations that result in coronary defects are due to failures in epicardial formation or loss of epicardial integrity, highlighting the critical role of the epicardium in this process. One exception to this trend was unveiled by genetic analysis of Fog-2. Mutations in Fog-2 (deletion or inability to interact with GATA4) led to severe defects in myocardial proliferation and coronary development despite a normal appearing epicardium (Tevosian et al. 2000; Crispino et al. 2001). These hearts failed to undergo epicardial EMT. This phenotype could be rescued by expression of Fog-2 in the myocardium, demonstrating that signals from the myocardium regulate epicardial EMT.

Interestingly, the myocardial hypoplasia and coronary defects seen in Fog-2 ${ }^{-/}$hearts are reminiscent of the phenotypes seen in hearts lacking myocardial FGF signaling. Given that FOG-2 activity in the myocardium regulates these processes, it is intriguing to speculate that FOG-2 and FGF signaling may be functionally related. One possibility is that FOG-2 may be necessary for the cardiomyoblast to receive FGF signaling. Consistently, several interactions between FGF and GATA signaling have been identified (Xu et al. 1999; Fossett and Schulz 2001; Bertrand et al. 2003; Iwahori et al. 2004; Murakami et al. 2004).

\section{A ventricular wave of $\mathrm{HH}$ activity mediates coronary development}

Previous work in avian systems has shown that coronary vessel development proceeds in a wave-like pattern, originating from the atrial-ventricular groove and extending toward the ventricular apices (Morabito et al. 2002; Reese et al. 2002; Wada et al. 2003). Strikingly, we found that $\mathrm{HH}$ signaling is activated in a similar pattern and is required for the wave-like growth of the coronary vascular plexus. Given that Shh expression does not appear to travel in a wave, the mechanism by which $\mathrm{HH}$ activation progresses toward the cardiac apex is unclear.

One possible mechanism by which a gradient of signaling can be formed in the face of uniform ligand expression is the presence of a competency gradient. That 
is, the target of signaling is not uniformly competent to receive or respond to the signal. In this model the heart would gain competence to receive $\mathrm{HH}$ signaling in a wave-like progression. Consistent with this notion, experiments describing the effects of myocardial GLI2 ${ }^{\star}$ expression revealed the presence of a competency gradient. Specifically, despite uniform CRE-mediated recombination of the transgene throughout the ventricle (absence of LacZ staining indicating recombination) (Fig. 8A,B), both Ptc1 and Vegf-A were only up-regulated within their normal areas of expression. Thus, even though constitutively active GLI2 was expressed throughout the ventricle, it only activated $\mathrm{HH}$ signaling (as reported by Ptc1) within its normal wave-like distribution.

\section{Subepicardial and intramyocardial blood vessels}

Through examination of normal coronary development in the mouse, we have identified two sets of coronary vessels: vessels growing in the subepicardial space, and vessels growing within the myocardial wall. We refer to these blood vessels as subepicardial and intramyocardial blood vessels, respectively. Both sets of coronary vessels grow in a wave-like progression and are regulated by FGF and $\mathrm{HH}$ signaling. Interestingly, growth of these vessels appeared to be regulated by $\mathrm{HH}$ signaling to different cell types, cardiomyoblasts and perivascular cells.

Experiments forcing $\mathrm{HH}$ activation in the myocardium indicated that $\mathrm{HH}$ signaling to the cardiomyoblast promotes subepicardial but not intramyocardial blood vessel growth. Together, these data and the fact that Vegf-C is expressed in cells surrounding intramyocardial blood vessels suggest that $\mathrm{HH}$ signaling to perivascular cells may specifically regulate intramyocardial blood vessel growth. Expression of Ptc1 in these cells is consistent with this hypothesis.

It is interesting to speculate that subepicardial and intramyocardial blood vessels may either differentially represent or give rise to distinct vessel types. Corrosion casting of developing coronary vessels in the rat demonstrated that veins grow within the subepicardial space while arteries grow within the myocardial wall (Ratajska et al. 2003). Given this, it is interesting to consider the possibility that subepicardial and intramyocardial blood vessels represent or give rise to veins and arteries, respectively. If this is true, coincident $\mathrm{HH}$ signaling to the cardiomyoblast and perivascular cell may coordinate venous and arterial growth. The ability of $\mathrm{HH}$ signaling to orchestrate balanced venous and arterial growth would have profound implications and applications for pharmacological treatment aimed at promoting coronary neovascularization.

\section{Materials and methods}

Embryonic heart organ culture

For slice cultures, E12.5 hearts were dissected under aseptic conditions and embedded in 5\% low-melt agar/PBS. Using a vibrotome, $150-\mu \mathrm{m}$ slices were obtained and placed on a Transwell filter over wells containing $1 \mathrm{~mL}$ of media (NMEM/10\% $\mathrm{FCS} / 2 \mu \mathrm{g} / \mathrm{mL}$ heparin). After incubating for $1 \mathrm{~h}\left(37^{\circ} \mathrm{C} / 5 \% \mathrm{CO}_{2}\right)$,
BSA, FGF9, or SHH protein \pm cyclopamine (see below) was added to the media and the explants were cultured at $37^{\circ} \mathrm{C} / 5 \%$ $\mathrm{CO}_{2}$. Organ cultures were harvested at $24 \mathrm{~h}$. The hearts were removed from the filters and fixed in either $4 \%$ PFA (in situ) or $2 \%$ GAD (LacZ staining).

For whole-heart cultures, E11.5 hearts were dissected under aseptic conditions and placed in glass scintillation vials containing $1 \mathrm{~mL}$ of media (above). Vehicle, cyclopamine, and/or protein was added to the vials. Caps were loosely screwed on and the vials were incubated for $24-48 \mathrm{~h}$ on a rocker at $37^{\circ} \mathrm{C} / 5 \%$ $\mathrm{CO}_{2}$. Hearts were harvested and fixed as above.

The following concentrations of protein were used: $0.1 \%$ BSA (Sigma), $100 \mathrm{ng} / \mu \mathrm{L}$ FGF9 (Peprotech), 50ng/mL VEGF-A ${ }_{165}$ (R\&D), $20 \mathrm{ng} / \mathrm{mL}$ ANG2 (R\&D), and $1 \mu \mathrm{g} / \mathrm{mL}$ SHH (R\&D). Cyclopamine was dissolved in ethanol and used at $10 \mu \mathrm{M}$. Vehicle was $1 \mu \mathrm{L} / \mathrm{mL}$ of ethanol.

RNA isolation, cDNA sysnthesis, and $q R T-P C R$ analysis

RNA was isolated from three biological samples (each sample consisted of four E13.5 ventricles) using the RNeasy kit (Qiagen). cDNA was obtained using the SuperScript II first-strand cDNA synthesis kit (Invitrogen).

qRT-PCR analysis was performed using SYBR green (Bio-Rad) and a Bio-Rad iCycler. The following primers were used: Vegf-A, Vegfr-2, Ang1, Ang2, and Pecam (Shih et al. 2002), Vegf-B (Mills et al. 2002), Vegf-C and Vegf-D (Chang et al. 2004), Ptc1 (Wang and Seed 2003), and Shh (Wang and Seed 2003) (forward, AAAGCTGACCCCTTTAGCCTA; reverse, TTCGGAGTTTC TTGTGATCTTCC). To control for the amount of input cDNA, all reactions were normalized to GAPDH. Results were graphed as relative expression compared with wild type, where wild type was scaled to 1. Error bars indicate standard deviation.

\section{Whole-mount in situ hybridization}

Whole-mount in situ hybridization was performed as previously described (Lavine et al. 2005). Tissues were photographed and then cryo-sectioned $(16 \mu \mathrm{m})$, mounted on slides, and rephotographed. In situ probes were as follows: Vegfr-1 andVegfr-2 (B. Olsen); Vegf-A (G. Karsenty); Vegf-B, Vegf-C, and Vegf-D (K. Alitalo); Ang1, Ang2, Tie1, and Tie2 (T. Sato); and Ptc1 and Shh (A. McMahon). All comparisons shown are between littermates, and all experiments were repeated at least three times.

\section{Whole-mount PECAM immunohistochemistry}

After fixation (4\% PFA), tissues were dehydrated in a methanol series, incubated in methanol/hydrogen peroxide, rehydrated, and blocked in PBSST ( $5 \%$ goat serum/PBS $0.1 \%$ Triton X-100). The primary antibody used was rat anti-mouse PECAM (R\&D, 1:200). Biotinylated goat anti-rat IgG (Vector, 1:200) was used, followed by Vectastain $\mathrm{ABC}$-peroxidase reagent and $\mathrm{DAB}$ visualization (Vector). All antibody and $\mathrm{ABC}$ reagent dilutions were done in PBSST. Antibody and $\mathrm{ABC}$ reagent incubations were carried out overnight at $4^{\circ} \mathrm{C}$. Following each overnight incubation, tissues were washed five times $\left(1 \mathrm{~h}\right.$ each at $\left.4^{\circ} \mathrm{C}\right)$ with PBSST.

After PECAM staining, hearts were photographed and analyzed using Canvas software. Quantitation of the percentage of the ventricle covered by blood vessels was performed by dividing the area covered by blood vessels by the total ventricular area. For each analysis at least three hearts were analyzed.

Following image analysis, PECAM-stained hearts were paraffin embedded and sectioned. Paraffin sections $(4 \mu \mathrm{m})$ were then dewaxed, rehydrated, counterstained with hematoxylin (Sigma), and mounted. 
Lavine et al.

\section{Florescent immunohistochemistry and LacZ staining}

For PECAM immunohistochemistry, paraffin sections (4 $\mu \mathrm{m})$ were dewaxed, rehydrated, and antigen-exposed with trypsin digestion. Following antigen exposure, sections were blocked in $10 \%$ goat serum/PBS and incubated with rat anti-mouse PECAM (R\&D, 1:100).

For SHH and PTC1 immunohistochemistry, 12- $\mu \mathrm{m}$ cryo-sections were cut from E12.5-E13.5 hearts and stained with SHH (Developmental Studies Hybridoma Bank, 5E1) 1:100, PTC1 (Santa Cruz, G-19) 1:100, PECAM (R\&D) 1:200, and cardiac actin (Sigma) 1:500.

LacZ staining was performed as described (Soriano 1999).

\section{Tomato lectin injection and visualization}

Biotinylated tomato lectin (Vector Labs, $1 \mathrm{mg} / \mathrm{mL}$ ) was injected into the inferior vena cava of anesthetized mice following $5 \mathrm{~d}$ of GLI2* induction. Five minutes after Lectin injection, hearts were dissected, fixed (10\% formalin), and embedded in paraffin. Paraffin sections $(4 \mu \mathrm{m})$ were then dewaxed, rehydrated, incubated in methanol/hydrogen peroxide, and blocked in $10 \%$ goat serum/PBS. Biotinylated lectin was visualized by staining with streptavidin-HRP (Vector Labs) and DAB (Vector Labs). Following staining, sections were counterstained with hematoxylin (Sigma) and mounted.

\section{Acknowledgments}

We thank $\mathrm{K}$. Yu and $\mathrm{H}$. Kanazawa for critically reading this manuscript, K. Chien for providing Mlc2v-Cre mice, R. Fassler for Tie1-Cre mice, J. Molkentin for aMHC-ER-Cre mice, and C. Hui for pCAGGs-LacZ ${ }^{\text {flox }}$-GLI2* mice. This work was initiated with a grant from the American Heart Association and funded by NIH grants HL076664, HD39952, and DK52574 (microinjection); American Heart Association grant 0415469Z; and a generous contribution from the Virginia Friedhofer Charitable Trust.

\section{References}

Aase, K., Lymboussaki, A., Kaipainen, A., Olofsson, B., Alitalo, K., and Eriksson, U. 1999. Localization of VEGF-B in the mouse embryo suggests a paracrine role of the growth factor in the developing vasculature. Dev. Dyn. 215: 12-25.

Auguste, P., Javerzat, S., and Bikfalvi, A. 2003. Regulation of vascular development by fibroblast growth factors. Cell Tissue Res. 314: 157-166.

Bellomo, D., Headrick, J.P., Silins, G.U., Paterson, C.A., Thomas, P.S., Gartside, M., Mould, A., Cahill, M.M., Tonks, I.D., Grimmond, S.M., et al. 2000. Mice lacking the vascular endothelial growth factor-B gene (Vegfb) have smaller hearts, dysfunctional coronary vasculature, and impaired recovery from cardiac ischemia. Circ. Res. 86: E29-E35.

Bertrand, V., Hudson, C., Caillol, D., Popovici, C., and Lemaire, P. 2003. Neural tissue in ascidian embryos is induced by FGF9/16/20, acting via a combination of maternal GATA and Ets transcription factors. Cell 115: 615-627.

Chang, L.K., Garcia-Cardena, G., Farnebo, F., Fannon, M., Chen, E.J., Butterfield, C., Moses, M.A., Mulligan, R.C., Folkman, J., and Kaipainen, A. 2004. Dose-dependent response of FGF-2 for lymphangiogenesis. Proc. Natl. Acad. Sci. 101: 11658-11663.

Chen, T.H., Chang, T.C., Kang, J.O., Choudhary, B., Makita, T., Tran, C.M., Burch, J.B., Eid, H., and Sucov, H.M. 2002. Epi- cardial induction of fetal cardiomyocyte proliferation via a retinoic acid-inducible trophic factor. Dev. Biol. 250: 198207.

Crispino, J.D., Lodish, M.B., Thurberg, B.L., Litovsky, S.H., Collins, T., Molkentin, J.D., and Orkin, S.H. 2001. Proper coronary vascular development and heart morphogenesis depend on interaction of GATA-4 with FOG cofactors. Genes \& Dev. 15: 839-844.

Davies, R., Moore, A., Schedl, A., Bratt, E., Miyahawa, K., Ladomery, M., Miles, C., Menke, A., van Heyningen, V., and Hastie, N. 1999. Multiple roles for the Wilms' tumor suppressor, WT1. Cancer Res. (7 Suppl) 59: 1747s-1750s. [Discussion 1751s]

Detillieux, K.A., Sheikh, F., Kardami, E., and Cattini, P.A. 2003. Biological activities of fibroblast growth factor- 2 in the adult myocardium. Cardiovasc. Res. 57: 8-19.

Dettman, R.W., Denetclaw Jr., W., Ordahl, C.P., and Bristow, J. 1998. Common epicardial origin of coronary vascular smooth muscle, perivascular fibroblasts, and intermyocardial fibroblasts in the avian heart. Dev. Biol. 193: 169-181.

Ferrara, N., Carver-Moore, K., Chen, H., Dowd, M., Lu, L., O'Shea, K.S., Powell-Braxton, L., Hillan, K.J., and Moore, M.W. 1996. Heterozygous embryonic lethality induced by targeted inactivation of the VEGF gene. Nature 380: 439442.

Fossett, N. and Schulz, R.A. 2001. Conserved cardiogenic functions of the multitype zinc-finger proteins: U-shaped and FOG-2. Trends Cardiovasc. Med. 11: 185-190.

Gale, N.W., Thurston, G., Hackett, S.F., Renard, R., Wang, Q., McClain, J., Martin, C., Witte, C., Witte, M.H., Jackson, D., et al. 2002. Angiopoietin-2 is required for postnatal angiogenesis and lymphatic patterning, and only the latter role is rescued by Angiopoietin-1. Dev. Cell 3: 411-423.

Gittenberger-de Groot, A.C., Vrancken Peeters, M.P., Bergwerff, M., Mentink, M.M., and Poelmann, R.E. 2000. Epicardial outgrowth inhibition leads to compensatory mesothelial outflow tract collar and abnormal cardiac septation and coronary formation. Circ. Res. 87: 969-971.

Gustafsson, E., Brakebusch, C., Hietanen, K., and Fassler, R. 2001. Tie-1-directed expression of Cre recombinase in endothelial cells of embryoid bodies and transgenic mice. J. Cell Sci. 114: 671-676.

Henry, G.D., Byrne, R., Hunyh, T.T., Abraham, V., Annex, B.H., Hagen, P.O., and Donatucci, C.F. 2000. Intracavernosal injections of vascular endothelial growth factor protects endothelial dependent corpora cavernosal smooth muscle relaxation in the hypercholesterolemic rabbit: A preliminary study. Int. J. Impot. Res. 12: 334-339.

Hiratsuka, S., Minowa, O., Kuno, J., Noda, T., and Shibuya, M. 1998. Flt-1 lacking the tyrosine kinase domain is sufficient for normal development and angiogenesis in mice. Proc. Nat1. Acad. Sci. 95: 9349-9354.

House, S.L., Bolte, C., Zhou, M., Doetschman, T., Klevitsky, R., Newman, G., and Schultz Jel, J. 2003. Cardiac-specific overexpression of fibroblast growth factor-2 protects against myocardial dysfunction and infarction in a murine model of low-flow ischemia. Circulation 108: 3140-3148.

Iwahori, A., Fraidenraich, D., and Basilico, C. 2004. A conserved enhancer element that drives FGF4 gene expression in the embryonic myotomes is synergistically activated by GATA and bHLH proteins. Dev. Biol. 270: 525-537.

Kanda, S., Mochizuki, Y., Suematsu, T., Miyata, Y., Nomata, K., and Kanetake, H. 2003. Sonic hedgehog induces capillary morphogenesis by endothelial cells through phosphoinositide 3-kinase. J. Biol. Chem. 278: 8244-8249.

Kanda, S., Miyata, Y., and Kanetake, H. 2004. Fibroblast growth 
factor-2-mediated capillary morphogenesis of endothelial cells requires signals via Flt-1/vascular endothelial growth factor receptor-1: Possible involvement of c-Akt. J. Biol. Chem. 279: 4007-4016.

Kusano, K.F., Pola, R., Murayama, T., Curry, C., Kawamoto, A., Iwakura, A., Shintani, S., Ii, M., Asai, J., Tkebuchava, T., et al. 2005. Sonic hedgehog myocardial gene therapy: Tissue repair through transient reconstitution of embryonic signaling. Nat. Med. 11: 1197-1204.

Kwee, L., Baldwin, H.S., Shen, H.M., Stewart, C.L., Buck, C., Buck, C.A., and Labow, M.A. 1995. Defective development of the embryonic and extraembryonic circulatory systems in vascular cell adhesion molecule (VCAM-1) deficient mice. Development 121: 489-503.

Landau, C., Jacobs, A.K., and Haudenschild, C.C. 1995. Intrapericardial basic fibroblast growth factor induces myocardial angiogenesis in a rabbit model of chronic ischemia. Am. Heart J. 129: 924-931.

Lavine, K.J., Yu, K., White, A.C., Zhang, X., Smith, C., Partanen, J., and Ornitz, D.M. 2005. Endocardial and epicardial derived FGF signals regulate myocardial proliferation and differentiation in vivo. Dev. Cell 8: 85-95.

Lewandoski, M., Sun, X., and Martin, G.R. 2000. Fgf8 signalling from the AER is essential for normal limb development. Nat. Genet. 26: 460-463.

Losordo, D.W., Vale, P.R., Hendel, R.C., Milliken, C.E., Fortuin, F.D., Cummings, N., Schatz, R.A., Asahara, T., Isner, J.M., and Kuntz, R.E. 2002. Phase $1 / 2$ placebo-controlled, doubleblind, dose-escalating trial of myocardial vascular endothelial growth factor 2 gene transfer by catheter delivery in patients with chronic myocardial ischemia. Circulation 105: 2012-2018.

Merki, E., Zamora, M., Raya, A., Kawakami, Y., Wang, J., Zhang, X., Burch, J., Kubalak, S.W., Kaliman, P., Belmonte, J.C., et al. 2005. Epicardial retinoid X receptor $\alpha$ is required for myocardial growth and coronary artery formation. Proc. Natl. Acad. Sci. 102: 18455-18460.

Mikawa, T. and Fischman, D.A. 1992. Retroviral analysis of cardiac morphogenesis: Discontinuous formation of coronary vessels. Proc. Nat1. Acad. Sci. 89: 9504-9508.

Mikawa, T. and Gourdie, R.G. 1996. Pericardial mesoderm generates a population of coronary smooth muscle cells migrating into the heart along with ingrowth of the epicardial organ. Dev. Biol. 174: 221-232.

Mill, P., Mo, R., Fu, H., Grachtchouk, M., Kim, P.C., Dlugosz, A.A., and Hui, C.C. 2003. Sonic hedgehog-dependent activation of Gli2 is essential for embryonic hair follicle development. Genes \& Dev. 17: 282-294.

Mills, J.C., Andersson, N., Hong, C.V., Stappenbeck, T.S., and Gordon, J.I. 2002. Molecular characterization of mouse gastric epithelial progenitor cells. Proc. Nat1. Acad. Sci. 99: 14819-14824.

Miquerol, L., Gertsenstein, M., Harpal, K., Rossant, J., and Nagy, A. 1999. Multiple developmental roles of VEGF suggested by a LacZ-tagged allele. Dev. Biol. 212: 307-322.

Moore, A.W., McInnes, L., Kreidberg, J., Hastie, N.D., and Schedl, A. 1999. YAC complementation shows a requirement for Wt1 in the development of epicardium, adrenal gland and throughout nephrogenesis. Development 126: $1845-1857$

Morabito, C.J., Kattan, J., and Bristow, J. 2002. Mechanisms of embryonic coronary artery development. Curr. Opin. Cardiol. 17: 235-241.

Motoike, T., Markham, D.W., Rossant, J., and Sato, T.N. 2003. Evidence for novel fate of $\mathrm{Flk} 1^{+}$progenitor: Contribution to muscle lineage. Genesis 35: 153-159.
Murakami, A., Shen, H., Ishida, S., and Dickson, C. 2004. SOX7 and GATA-4 are competitive activators of Fgf-3 transcription. J. Biol. Chem. 279: 28564-28573.

Partanen, T.A., Makinen, T., Arola, J., Suda, T., Weich, H.A., and Alitalo, K. 1999. Endothelial growth factor receptors in human fetal heart. Circulation 100: 583-586.

Pearse II, R.V., Vogan, K.J., and Tabin, C.J. 2001. Ptc1 and Ptc2 transcripts provide distinct readouts of Hedgehog signaling activity during chick embryogenesis. Dev. Biol. 239: 15-29.

Perez-Pomares, J.M., Carmona, R., Gonzalez-Iriarte, M., Atencia, G., Wessels, A., and Munoz-Chapuli, R. 2002. Origin of coronary endothelial cells from epicardial mesothelium in avian embryos. Int. J. Dev. Biol. 46: 1005-1013.

Pola, R., Ling, L.E., Silver, M., Corbley, M.J., Kearney, M., Blake Pepinsky, R., Shapiro, R., Taylor, F.R., Baker, D.P., Asahara, T., et al. 2001. The morphogen Sonic hedgehog is an indirect angiogenic agent upregulating two families of angiogenic growth factors. Nat. Med. 7: 706-711.

Puri, M.C., Partanen, J., Rossant, J., and Bernstein, A. 1999. Interaction of the TEK and TIE receptor tyrosine kinases during cardiovascular development. Development 126: 4569-4580.

Rajanayagam, M.A., Shou, M., Thirumurti, V., Lazarous, D.F., Quyyumi, A.A., Goncalves, L., Stiber, J., Epstein, S.E., and Unger, E.F. 2000. Intracoronary basic fibroblast growth factor enhances myocardial collateral perfusion in dogs. J. Am. Coll. Cardiol. 35: 519-526.

Ratajska, A., Ciszek, B., and Sowinska, A. 2003. Embryonic development of coronary vasculature in rats: Corrosion casting studies. Anat. Rec. A Discov. Mol. Cell. Evol. Biol. 270: 109116.

Reese, D.E., Mikawa, T., and Bader, D.M. 2002. Development of the coronary vessel system. Circ. Res. 91: 761-768.

Scheinowitz, M., Abramov, D., and Eldar, M. 1997. The role of insulin-like and basic fibroblast growth factors on ischemic and infarcted myocardium: A mini review. Int. J. Cardiol. 59: $1-5$.

Seghezzi, G., Patel, S., Ren, C.J., Gualandris, A., Pintucci, G., Robbins, E.S., Shapiro, R.L., Galloway, A.C., Rifkin, D.B., and Mignatti, P. 1998. Fibroblast growth factor-2 (FGF-2) induces vascular endothelial growth factor (VEGF) expression in the endothelial cells of forming capillaries: An autocrine mechanism contributing to angiogenesis. J. Cell Biol. 141: 1659-1673.

Shalaby, F., Rossant, J., Yamaguchi, T.P., Gertsenstein, M., Wu, X.F., Breitman, M.L., and Schuh, A.C. 1995. Failure of bloodisland formation and vasculogenesis in Flk-1-deficient mice. Nature 376: 62-66.

Shao, Y., Lu, J., Zhang, G., Liu, C., and Huang, B. 2005. Histone acetyltransferase p300 promotes the activation of human WT1 promoter and intronic enhancer. Arch. Biochem. Biophys. 436: 62-68.

Shih, S.C., Robinson, G.S., Perruzzi, C.A., Calvo, A., Desai, K., Green, J.E., Ali, I.U., Smith, L.E., and Senger, D.R. 2002. Molecular profiling of angiogenesis markers. Am. J. Pathol. 161: 35-41.

Shikama, N., Lutz, W., Kretzschmar, R., Sauter, N., Roth, J.F., Marino, S., Wittwer, J., Scheidweiler, A., and Eckner, R. 2003. Essential function of p300 acetyltransferase activity in heart, lung and small intestine formation. EMBO J. 22: 51755185.

Simons, M., Annex, B.H., Laham, R.J., Kleiman, N., Henry, T., Dauerman, H., Udelson, J.E., Gervino, E.V., Pike, M., Whitehouse, M.J., et al. 2002. Pharmacological treatment of coronary artery disease with recombinant fibroblast growth factor-2: Double-blind, randomized, controlled clinical trial. 
Circulation 105: 788-793.

Sohal, D.S., Nghiem, M., Crackower, M.A., Witt, S.A., Kimball, T.R., Tymitz, K.M., Penninger, J.M., and Molkentin, J.D. 2001. Temporally regulated and tissue-specific gene manipulations in the adult and embryonic heart using a tamoxifeninducible Cre protein. Circ. Res. 89: 20-25.

Soriano, P. 1999. Generalized lacZ expression with the ROSA26 Cre reporter strain. Nat. Genet. 21: 70-71.

Stuckmann, I., Evans, S., and Lassar, A.B. 2003. Erythropoietin and retinoic acid, secreted from the epicardium, are required for cardiac myocyte proliferation. Dev. Biol. 255: 334-349.

Sucov, H.M., Dyson, E., Gumeringer, C.L., Price, J., Chien, K.R., and Evans, R.M. 1994. RXR amutant mice establish a genetic basis for vitamin A signaling in heart morphogenesis. Genes \& Dev. 8: 1007-1018.

Syed, I.S., Sanborn, T.A., and Rosengart, T.K. 2004. Therapeutic angiogenesis: A biologic bypass. Cardiology 101: 131-143.

Tammela, T., Enholm, B., Alitalo, K., and Paavonen, K. 2005. The biology of vascular endothelial growth factors. Cardiovasc. Res. 65: 550-563.

Tevosian, S.G., Deconinck, A.E., Tanaka, M., Schinke, M., Litovsky, S.H., Izumo, S., Fujiwara, Y., and Orkin, S.H. 2000. FOG-2, a cofactor for GATA transcription factors, is essential for heart morphogenesis and development of coronary vessels from epicardium. Cell 101: 729-739.

Tomanek, R.J., Holifield, J.S., Reiter, R.S., Sandra, A., and Lin, J.J. 2002. Role of VEGF family members and receptors in coronary vessel formation. Dev. Dyn. 225: 233-240.

Uchida, Y., Yanagisawa-Miwa, A., Nakamura, F., Yamada, K., Tomaru, T., Kimura, K., and Morita, T. 1995. Angiogenic therapy of acute myocardial infarction by intrapericardial injection of basic fibroblast growth factor and heparin sulfate: An experimental study. Am. Heart J. 130: 1182-1188.

Visconti, R.P., Richardson, C.D., and Sato, T.N. 2002. Orchestration of angiogenesis and arteriovenous contribution by angiopoietins and vascular endothelial growth factor (VEGF). Proc. Nat1. Acad. Sci. 99: 8219-8224.

Vokes, S.A., Yatskievych, T.A., Heimark, R.L., McMahon, J., McMahon, A.P., Antin, P.B., and Krieg, P.A. 2004. Hedgehog signaling is essential for endothelial tube formation during vasculogenesis. Development 131: 4371-4380.

Vrancken Peeters, M.P., Gittenberger-de Groot, A.C., Mentink, M.M., and Poelmann, R.E. 1999. Smooth muscle cells and fibroblasts of the coronary arteries derive from epithelialmesenchymal transformation of the epicardium. Anat. Embryol. (Berl.) 199: 367-378.

Wada, A.M., Willet, S.G., and Bader, D. 2003. Coronary vessel development: A unique form of vasculogenesis. Arterioscler. Thromb. Vasc. Biol. 23: 2138-2145.

Wang, X. and Seed, B. 2003. A PCR primer bank for quantitative gene expression analysis. Nucleic Acids Res. 31: e154.

Ward, N.L. and Dumont, D.J. 2002. The angiopoietins and Tie2/ Tek: Adding to the complexity of cardiovascular development. Semin. Cell Dev. Biol. 13: 19-27.

Ward, N.L., Van Slyke, P., and Dumont, D.J. 2004. Functional inhibition of secreted angiopoietin: A novel role for angiopoietin 1 in coronary vessel patterning. Biochem. Biophys. Res. Commun. 323: 937-946.

Watt, A.J., Battle, M.A., Li, J., and Duncan, S.A. 2004. GATA4 is essential for formation of the proepicardium and regulates cardiogenesis. Proc. Natl. Acad. Sci. 101: 12573-12578.

White, A.C., Xu, J., Yin, Y., Smith, C., Schmid, G., and Ornitz, D.M. 2006. FGF9 and SHH signaling coordinate lung growth and development through regulation of distinct mesenchymal domains. Development 133: 1507-1517.

Xu, R.H., Ault, K.T., Kim, J., Park, M.J., Hwang, Y.S., Peng, Y.,
Sredni, D., and Kung, H. 1999. Opposite effects of FGF and BMP-4 on embryonic blood formation: Roles of PV.1 and GATA-2. Dev. Biol. 208: 352-361.

Yang, J.T., Rayburn, H., and Hynes, R.O. 1995. Cell adhesion events mediated by $\alpha 4$ integrins are essential in placental and cardiac development. Development 121: 549-560. 


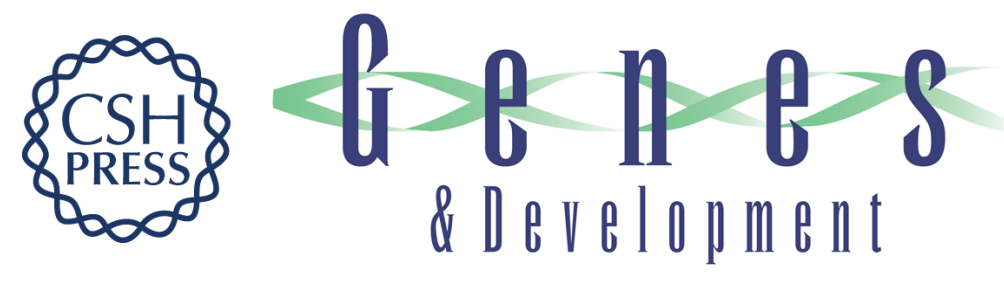

\section{Fibroblast growth factor signals regulate a wave of Hedgehog activation that is essential for coronary vascular development}

Kory J. Lavine, Andrew C. White, Changwon Park, et al.

Genes Dev. 2006, 20:

Access the most recent version at doi:10.1101/gad.1411406

Supplemental http://genesdev.cshlp.org/content/suppl/2006/06/01/20.12.1651.DC1
Material

References This article cites 72 articles, 30 of which can be accessed free at:

http://genesdev.cshlp.org/content/20/12/1651.full.html\#ref-list-1

License

Email Alerting Receive free email alerts when new articles cite this article - sign up in the box at the top

Service

right corner of the article or click here.

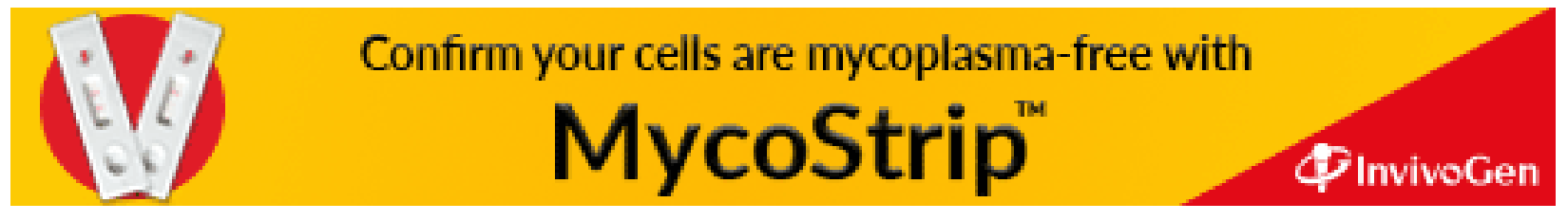

